\title{
Merit functions: a bridge between optimization and equilibria
}

\author{
Massimo Pappalardo* Giandomenico Mastroeni* Mauro Passacantando*
}

\begin{abstract}
In the last decades, many problems involving equilibria, arising from engineering, physics and economics, have been formulated as variational mathematical models. In turn, these models can be reformulated as optimization problems through merit functions. This paper aims at reviewing the literature about merit functions for variational inequalities, quasi-variational inequalities and abstract equilibrium problems. Smoothness and convexity properties of merit functions and solution methods based on them will be presented.
\end{abstract}

Keywords. Merit functions, gap functions, variational inequalities, equilibrium problems, descent methods.

\section{Introduction}

Optimization is a widespread mathematical technique in many engineering and economic applications. However, in many real-world problems, an objective function to be optimized is missing and the concept of equilibrium becomes crucial. Roughly speaking, if optimization takes care of the system utility function, the equilibrium takes into account the mutual interaction between users. In recent years, the interest in equilibrium problems has widely grown. The main applications are concerned with traffic over telecommunication networks or over public roads, oligopolistic and spatial price markets, financial markets, risk management, climate competition, migration problems, power allocation in radio systems, internet advertising, cloud computing (see e.g. $[3,5,6,13,16,24,28,34,36,48,49,50,54,66,68,70,71,80,76,88,95,102]$ and references therein).

All these problems have been formulated in the literature through variational mathematical models as complementarity problems, variational inequalities, quasi-variational inequalities, and Nash equilibrium problems among others. Variational inequalities (VIs) are one of the most known variational models. They were introduced by Hartman and Stampacchia [44] as a tool for studying partial differential equations in infinite dimensional spaces arising from mechanics (free-obstacle problem, friction problem, etc.). Later, their applications to contact problems in mechanical structures provided a vaste source of finite dimensional problems.

A finite-dimensional VI is defined as follows:

$$
\text { find } x^{*} \in C \text { such that }\left\langle F\left(x^{*}\right), y-x^{*}\right\rangle \geq 0 \text {, for all } y \in C,
$$

\footnotetext{
*Dipartimento di Informatica, Università di Pisa, Largo B. Pontecorvo 3, 56127 Pisa, Italy.
} 
where $F: \mathbb{R}^{n} \rightarrow \mathbb{R}^{n}, C$ is a closed and convex subset of $\mathbb{R}^{n}$ and $\langle\cdot, \cdot\rangle$ is the scalar product in $\mathbb{R}^{n}$.

Several kinds of numerical methods to solve VIs have been proposed (see, e.g., [33, 42] and references therein). One popular approach is based on the reformulation of (VI) as an optimization problem through suitable merit functions.

A function $p: \mathbb{R}^{n} \rightarrow \mathbb{R}$ is called merit function for (VI) if there exists a set $\Omega \subseteq \mathbb{R}^{n}$ such that:

- $p$ is nonnegative on $\Omega$,

- $x^{*}$ is a solution to (VI) if and only if $x^{*} \in \Omega$ and $p\left(x^{*}\right)=0$.

If the set $\Omega$ coincides with the feasible set $C$ of (VI), a merit function is also known in the literature as a gap function. Therefore, merit functions are the key concept to build a bridge between VIs and optimization.

In this paper we aim at reviewing the state of the art concerning the merit function approach for VIs and two interesting generalization of VIs: quasi-variational inequalities and abstract equilibrium problems.

The rest of paper is organized as follows: Section 2 is devoted to the preliminary concepts that will be used in the paper. Sections 3 deals with both constrained and unconstrained optimization reformulations of (VI). In particular, we will describe continuity and differentiability properties of merit functions, conditions under which merit functions are convex or their stationary points solve (VI), and error bound results, i.e., how the distance between an arbitrary point $x$ and the solution set of (VI) can be estimated in terms of the merit function value at $x$. Furthermore, $a d-$ hoc descent methods for minimizing merit functions will be shown. Section 4 and 5 are devoted to the results about merit functions for quasi-variational inequalities and abstract equilibrium problems, respectively. Examples of applications of the presented models are provided in Sections 3, 4 and 5. Some concluding remarks and suggestions for future research are collected in Section 6. We hope that this paper may stimulate further interest in merit functions and may be the basis to obtain new results.

\section{Preliminaries}

In this section, we show two particular cases of (VI), and we recall the main definitions and preliminary results that will be used throughout the paper. We make the blanket assumptions that the feasible set $C$ of (VI) is closed and convex and the operator $F$ is continuous on $C$.

Optimality conditions. As first particular case, let us consider the problem of finding a local minimum $x^{*}$ of a differentiable function $\psi: \mathbb{R}^{n} \rightarrow \mathbb{R}$ over the set $C$. The classic first order necessary optimality condition states that the directional derivative of $\psi$ at $x^{*}$ in any feasible direction is nonnegative, i.e.

$$
\left\langle\nabla \psi\left(x^{*}\right), y-x^{*}\right\rangle \geq 0, \quad \forall y \in C .
$$

This condition is a particular case of (VI) where $F(x)=\nabla \psi(x)$.

Complementarity problems. Another example of (VI) is provided by a complementarity problem described as follows: given a closed convex cone $C \subseteq \mathbb{R}^{n}$ and a mapping $F: \mathbb{R}^{n} \rightarrow \mathbb{R}^{n}$, the complementarity problem asks to determine a point $x^{*} \in C$ such that

$$
\left\langle F\left(x^{*}\right), x^{*}\right\rangle=0 \quad \text { and } \quad F\left(x^{*}\right) \in C^{*},
$$


where $C^{*}$ denotes the dual cone of $C$, i.e.

$$
C^{*}:=\left\{d \in \mathbb{R}^{n}:\langle d, y\rangle \geq 0 \text { for all } y \in C\right\} .
$$

Solving the complementarity problem amounts to solving (VI). In fact, if $x^{*}$ solves the complementarity problem, then for any $y \in C$ we have

$$
\left\langle F\left(x^{*}\right), y-x^{*}\right\rangle=\left\langle F\left(x^{*}\right), y\right\rangle \geq 0,
$$

hence $x^{*}$ solves (VI); vice versa, if $x^{*}$ solves (VI), then setting $y=0$ and $y=2 x^{*}$ (which belong to $C$ because $C$ is a cone) we obtain $\left\langle F\left(x^{*}\right), x^{*}\right\rangle=0$ and hence $F\left(x^{*}\right) \in C^{*}$, that is $x^{*}$ is a solution to the complementarity problem. Note that if we define

$$
p(x):=\langle F(x), x\rangle, \quad \Omega:=\left\{x \in C: F(x) \in C^{*}\right\},
$$

then $p(x) \geq 0$ for any $x \in \Omega$ and $x^{*}$ solves the complementarity problem if and only if $x^{*} \in \Omega$ and $p\left(x^{*}\right)=0$, i.e. $p$ is a merit function for the complementarity problem.

Monotonicity definitions. Monotonicity is a key assumption to establish existence of solutions, convergence results for algorithms and to provide error bounds for (VI). We now recall the main monotonicity properties that will be exploited in the paper. $F$ is said monotone on $C$ if

$$
\langle F(x)-F(y), x-y\rangle \geq 0, \quad \forall x, y \in C ;
$$

the corresponding concept of strict monotonicity is analogously defined just requiring strict inequality to hold for any $x, y \in C$ with $x \neq y ; F$ is said strongly monotone on $C$ with modulus $\mu$ if

$$
\langle F(x)-F(y), x-y\rangle \geq \mu\|x-y\|^{2}, \quad \forall x, y \in C,
$$

for some $\mu>0 ; F$ is said pseudomonotone on $C$ if for any $x, y \in C$ one has

$$
\langle F(y), x-y\rangle \geq 0 \quad \Longrightarrow \quad\langle F(x), x-y\rangle \geq 0 .
$$

In the particular case where $F(x)=\nabla \psi(x)$, monotonicity and strong monotonicity of $F$ on $C$ are equivalent to convexity and strong convexity of $\psi$ on $C$, respectively.

Existence results. We now recall two basic results concerning the existence of a solution to (VI). For the sake of simplicity, we will not consider the sharpest possible assumptions. The solution set of (VI) is nonempty if either the feasible set $C$ is bounded [44] or the following coercivity condition holds: there exists a point $y \in C$ such that

$$
\lim _{\|x\| \rightarrow \infty, x \in C}\langle F(x), y-x\rangle=-\infty .
$$

In particular, condition (2) holds if $F$ is strongly monotone on $C$. Moreover, the strong monotonicity of $F$ ensures that (VI) has a unique solution.

Fixed point problem reformulation. If we denote by $\pi_{C}$ the Euclidean projection operator on $C$, i.e.,

$$
\pi_{C}(x):=\arg \min _{y \in C}\|y-x\|,
$$

then it is well-known that (VI) is equivalent to finding a fixed point of the operator $x \mapsto \pi_{C}(x-$ $F(x))$. 
Complementarity problem reformulation. Assuming

$$
C:=\left\{x \in \mathbb{R}^{n}: g_{i}(x) \leq 0, \quad i=1, \ldots, m\right\},
$$

where the functions $g_{i}$ are differentiable and convex for all $i=1, \ldots, m$, it is possible to derive the Karush-Kuhn-Tucker (KKT) conditions for (VI). In fact, $x^{*}$ is a solution to (VI) if and only if it is a global minimum of the following convex optimization problem:

$$
\min _{y \in C}\left\langle F\left(x^{*}\right), y\right\rangle \text {. }
$$

Under some constraint qualification, the following KKT conditions

$$
\begin{cases}F\left(x^{*}\right)+\sum_{i=1}^{m} \lambda_{i}^{*} \nabla g_{i}\left(x^{*}\right)=0, & \\ \lambda_{i}^{*} g_{i}\left(x^{*}\right)=0, & i=1, \ldots, m, \\ \lambda_{i}^{*} \geq 0, \quad g_{i}\left(x^{*}\right) \leq 0, & i=1, \ldots, m,\end{cases}
$$

are necessary and sufficient for optimality and, in turn, for the existence of solutions to (VI). It is well-known that KKT system (4) is equivalent to a complementarity problem.

\section{Merit functions for variational inequalities}

In this section we summarize several approaches in order to express (VI) as a constrained or unconstrained optimization problem by means of different merit functions.

A first merit function can be defined by exploiting the fixed point reformulation stated in Section 2. In fact, the function $x \mapsto\left\|x-\pi_{C}(x-F(x))\right\|$ is a gap function for (VI) [29].

A further merit function for (VI) can be obtained by means of the complementarity reformulation (4), which in turn can be associated with the merit function (1). Further examples of merit functions associated with a complementarity problem can be found in [35].

\subsection{Constrained optimization reformulations}

This section is devoted to gap functions for (VI).

\subsubsection{Auslender gap function}

A first example of gap function was given in [8], where the following function was introduced:

$$
p(x):=\sup _{y \in C}\langle F(x), x-y\rangle \text {. }
$$

It is trivial to prove that $p$ is a gap function for (VI). Since the supremum in (5) can be infinite or not attained in a unique point, this function is in general neither finite, nor differentiable, nor convex. However, when $C$ is bounded and $F$ is continuously differentiable, it is finite and admits directional derivatives $p^{\prime}(x ; d)$ at any point $x \in C$ in any direction $d$. Moreover, if $F$ is monotone, any stationary point $x^{*}$ of $p$ on $C$, i.e.

$$
p^{\prime}\left(x^{*} ; y-x^{*}\right) \geq 0, \quad \forall y \in C,
$$


is a solution to (VI). In the particular case of monotone affine VIs, i.e. if $F(x)=A x+b$ where $A$ is a positive semidefinite matrix, $p$ also turns out to be convex [58]. If $F$ is strongly monotone with modulus $\mu$ and $x^{*}$ is the unique solution to (VI), then $p$ provides the following error bound:

$$
\left\|x-x^{*}\right\| \leq \sqrt{p(x) / \mu}, \quad \forall x \in C .
$$

A descent method based on the function $p$ has been proposed in [61] in the case where $C$ is a bounded polyhedron. At each iteration, the descent direction is obtained by minimizing a linearization of a further gap function. If $F$ is monotone, then the algorithm is globally convergent $^{1}$ to a solution to (VI). Moreover, the convergence is quadratic ${ }^{2}$ if $F$ is strongly monotone and the termination is achieved in a finite number of iterations if $F$ is affine. Under a so-called "geometric stability condition", it is shown that $p$ also provides an error bound for (VI).

\subsubsection{Regularized gap functions}

Many efforts of the research have been directed to the study of differentiable gap functions in order to simplify the computational aspects of the problem. Important results in this sense have been obtained in $[37,53,96,104]$.

First, Auchmuty [7] proposed a scheme in order to define a general class of gap functions:

$$
p^{A}(x):=\sup _{y \in C}[\langle F(x), x-y\rangle+f(x)-f(y)-\langle\nabla f(x), x-y\rangle],
$$

where $f: \mathbb{R}^{n} \rightarrow \mathbb{R}$ is convex and continuously differentiable. It was proved that if $\left(x^{*}, y^{*}\right)$ is a saddle point of the function

$$
L(x, y):=\langle F(x), x-y\rangle+f(x)-f(y)-\langle\nabla f(x), x-y\rangle
$$

on $C \times C$, i.e.,

$$
L\left(x^{*}, y\right) \leq L\left(x^{*}, y^{*}\right) \leq L\left(x, y^{*}\right), \quad \forall(x, y) \in C \times C,
$$

then $x^{*}$ is a solution to (VI) and $p^{A}$ is a gap function. We observe that if $f$ is strongly convex on $C$ and $F$ is differentiable, then the function $p^{A}$ is finite and differentiable.

Later, Fukushima [37] introduced a gap function which is a special case of (6), setting $f(x)=$ $\langle x, M x\rangle / 2$, where $M$ is a symmetric and positive definite matrix. It is defined by

$$
p^{F}(x):=\max _{y \in C}\left[\langle F(x), x-y\rangle-\frac{1}{2}\langle x-y, M(x-y)\rangle\right] .
$$

Note that the maximum in (7) is always attained in a unique point $y(x)$ since the objective function is strongly concave with respect to the variable $y$, hence $p^{F}$ is always finite. If $F$ is continuously differentiable, then Danskin's theorem [25] guarantees that also $p^{F}$ is so and

$$
\nabla p^{F}(x)=F(x)-\left[(\nabla F(x))^{T}-M\right](y(x)-x) .
$$

\footnotetext{
${ }^{1}$ The convergence is said global if it does not depend on the choice of the starting point.

${ }^{2} \mathrm{~A}$ sequence $\left\{x^{k}\right\}$ is said to be convergent to $\bar{x}$ with rate of convergence equal to $r$ if

$$
\limsup _{k \rightarrow+\infty} \frac{\left\|x^{k+1}-\bar{x}\right\|}{\left\|x^{k}-\bar{x}\right\|^{r}}=\gamma \in(0,+\infty) .
$$

If $r=1$ and $\gamma \in(0,1)$, then the convergence is said to be linear, if $r>1$, then the convergence is said to be superlinear, and, in particular, if $r=2$, the convergence is said to be quadratic.
} 
Moreover, if $x^{*}$ is a stationary point of $p^{F}$ on $C$, i.e.

$$
\left\langle\nabla p^{F}\left(x^{*}\right), y-x^{*}\right\rangle \geq 0, \quad \forall y \in C,
$$

and the Jacobian matrix $\nabla F\left(x^{*}\right)$ is positive definite, then $x^{*}$ is a solution to (VI). In the special case of strongly monotone affine VIs, i.e. $F(x)=A x+b$ with $A$ positive definite, $p^{F}$ turns out to be convex (strongly convex) provided that the matrix $A+A^{T}-M$ is positive semidefinite (positive definite) [53].

A descent algorithm for minimizing $p^{F}$ has been proposed in [37]: given any starting point $x^{0} \in C$, the sequence $\left\{x^{k}\right\}$ is generated by the iterations

$$
x^{k+1}=x^{k}+t_{k} d^{k}
$$

where the search direction $d^{k}=y\left(x^{k}\right)-x^{k}$ and the stepsize $t_{k} \in(0,1]$ is such that

$$
p^{F}\left(x^{k}+t_{k} d^{k}\right)=\min _{t \in(0,1]} p^{F}\left(x^{k}+t d^{k}\right) .
$$

Under the assumptions that $C$ is bounded, $F$ is continuously differentiable on $C$ and $\nabla F(x)$ is positive definite for all $x \in C$, the sequence $\left\{x^{k}\right\}$ belongs to $C$ and converges to the unique solution to (VI). This algorithm converges also employing an inexact line search rule, provided that $F$ is strongly monotone on $C$ and $\nabla F$ is Lipschitz continuous on $C$.

A variant of the above method which does not require the strong monotonicity of $F$ has been proposed in [103], setting the matrix $M=\alpha I$, where $I$ is the identity matrix. In fact, the monotonicity of $F$ paired with the boundedness of $C$ guarantees that at any point $x \in C$ the vector $y(x)-x$, which depends on the matrix $M$ and hence depends on $\alpha$, is a descent direction for $p^{F}$, provided that $\alpha$ is small enough. The method performs a line search at the current iterate $x^{k}$ if $y\left(x^{k}\right)-x^{k}$ is a descent direction for $p^{F}$; otherwise the value of $\alpha$ is decreased. The method is globally convergent if $C$ is bounded and $F$ is continuously differentiable and monotone on $C$. This algorithm has been extended to nonsmooth VIs in [79].

In $[60,93]$ the authors propose a modified Newton method: at each iteration it finds the solution to the linearized (VI) at $x$, i.e.

$$
\text { find } z(x) \text { s.t. }\langle F(x)+\nabla F(x)[z(x)-x], y-z(x)\rangle \geq 0, \quad \forall y \in C \text {. }
$$

In the hypothesis of strong monotonicity of $F$, problem (10) admits a unique solution $z(x)$ such that $d=z(x)-x$ is a descent direction for the gap functions $p^{A}$ and $p^{F}$ : the function $p^{A}$ has been considered in [60], while $p^{F}$ in [93]. By employing a line search strategy, the method is shown to be quadratically convergent under suitable additional assumptions.

When the feasible set $C$, defined as in (3), is not a polyhedron, the evaluation of the regularized gap function $p^{F}$ at a given point $x$ could be computationally expensive. In order to overcome this drawback, the following gap function has been proposed [92]:

$$
p^{T F}(x):=\max _{y \in T(x)}\left[\langle F(x), x-y\rangle-\frac{1}{2}\langle x-y, M(x-y)\rangle\right],
$$

where

$$
T(x):=\left\{y \in \mathbb{R}^{n}: g_{i}(x)+\left\langle\nabla g_{i}(x), y-x\right\rangle \leq 0, \quad i=1, \ldots, m\right\}
$$


is an outer polyhedral approximation of $C$ at $x$. If $F$ is continuously differentiable, $g_{i}$ 's are continuously differentiable and a constraint qualification holds, then $p^{T F}$ is directionally differentiable. Furthermore, if $\nabla F$ is positive definite and $g_{i}$ 's are twice continuously differentiable, then any stationary point of $p^{T F}$ on $C$ is a solution to (VI). A successive quadratic programming algorithm based on the minimization of an exact penalty function associated with $p^{T F}$ has been proposed in [92].

A generalization of the gap function introduced by Fukushima has been proposed in [96, 104] by replacing in (7) the regularizing term $\langle x-y, M(x-y)\rangle / 2$ with a general bifunction $G$ : $\mathbb{R}^{n} \times \mathbb{R}^{n} \rightarrow \mathbb{R}$ such that:

$$
\begin{aligned}
& G(x, y) \geq 0 \text { for all }(x, y) \in \mathbb{R}^{n} \times \mathbb{R}^{n}, \\
& G \text { is continuously differentiable, } \\
& G(x, \cdot) \text { is strongly convex on } C \text { for all } x \in C, \\
& G(x, x)=\nabla_{y} G(x, x)=0 \text { for all } x \in C .
\end{aligned}
$$

For any $\alpha>0$, the function

$$
p_{\alpha}(x):=\max _{y \in C}[\langle F(x), x-y\rangle-\alpha G(x, y)]
$$

turns out to be a gap function, which is continuously differentiable, if $F$ is so, with

$$
\nabla p_{\alpha}(x)=F(x)-(\nabla F(x))^{T}\left(y_{\alpha}(x)-x\right)-\alpha \nabla_{x} G\left(x, y_{\alpha}(x)\right),
$$

where $y_{\alpha}(x)$ is the unique solution to problem (14). Note that when $F$ is only locally Lipschitz continuous, the function $p_{\alpha}$ is also locally Lipschitz and its Clarke generalized gradient satisfies a formula similar to (15) (see [73]). Moreover, if $x^{*}$ is a stationary point of $p_{\alpha}$ on $C$ and $\nabla F\left(x^{*}\right)$ is positive definite, then $x^{*}$ solves (VI). A further important feature of $p_{\alpha}$ is that, under the assumption that $F$ is strongly monotone and $\nabla_{y} G(x, \cdot)$ is Lipschitz continuous on $C$, for every $x \in C$, it provides an error bound [104], i.e., there exists $M>0$ such that

$$
\left\|x-x^{*}\right\| \leq M \sqrt{p_{\alpha}(x)}, \quad \forall x \in C,
$$

where $x^{*}$ is the unique solution to (VI). In particular, (16) implies the boundedness of the sublevel sets of $p_{\alpha}$, which is of crucial importance in the convergence of the minimization algorithms.

The descent methods developed in [37] have been generalized to a more general framework exploiting several classes of gap functions defined by (14) (see [104]). Given a continuous mapping $\Gamma: C \times C \rightarrow \mathbb{R}^{n}$, such that $\Gamma(x, \cdot)$ is strongly monotone on $C$ for any $x \in C$, the following auxiliary variational inequality is considered at a given point $x \in C$ : find $y^{*} \in C$ such that

$$
\left\langle\Gamma\left(x, y^{*}\right)-\Gamma(x, x)+F(x), y-y^{*}\right\rangle \geq 0, \quad \forall y \in C .
$$

Having denoted by $w(x)$ the unique solution to $(\operatorname{AVI}(x))$, one can prove that the mapping $w: C \rightarrow C$ is continuous and $x^{*}$ is a solution to (VI) if and only if $x^{*}=w\left(x^{*}\right)$. In view of this result the following iterative method is proposed. Given $x^{k} \in C$, compute $w\left(x^{k}\right)$ : if $w\left(x^{k}\right)=x^{k}$, then $x^{k}$ is a solution to (VI), otherwise find $x^{k+1}$ performing an Armijo ${ }^{3}$ inexact line search for the gap function $p_{\alpha}$ along the direction $d^{k}:=w\left(x^{k}\right)-x^{k}$.

\footnotetext{
${ }^{3}$ The Armijo inexact line search along the direction $d^{k}$ consists in finding the smallest non negative integer $m$ such that

$$
p_{\alpha}\left(x^{k}+\beta^{m} d^{k}\right) \leq p_{\alpha}\left(x^{k}\right)-\sigma \beta^{m}\left\|d^{k}\right\|^{2},
$$

where $\beta, \sigma \in(0,1)$ are parameters, and then setting $x^{k+1}:=x^{k}+\beta^{m} d^{k}$.
} 
Each combination of $G$ and $\Gamma$ generates a different descent algorithm, which is globally convergent to the unique solution to (VI) under the assumption of continuous differentiability and strong monotonicity of $F$ and suitable additional assumptions on $G$ and $\Gamma$ [104]. Note that the algorithm (8)-(9) previously described is recovered by setting $\Gamma(x, y)=M(y-x)$.

The analysis of the convergence properties of the descent methods based on the regularized gap functions $p^{F}$ and $p_{\alpha}$ in the case where the operator $F$ is nondifferentiable has been considered in $[72,94]$.

\subsubsection{Minty (dual) gap functions}

The Minty (or dual) variational inequality was introduced in [67] and consists in finding $x^{*} \in C$ such that

$$
\left\langle F(y), y-x^{*}\right\rangle \geq 0, \quad \forall y \in C .
$$

Its relevance to applications was pointed out in $[40,77,78]$. In particular, Minty states the equivalence between (VI) and (MVI) when $F$ is pseudomonotone on $C$ [67].

In parallel with the Auslender gap function, it can be shown that

$$
p^{M}(x):=\sup _{y \in C}\langle F(y), x-y\rangle
$$

is a gap function for (MVI) and hence it is a gap function for (VI) provided that $F$ is pseudomonotone on $C$.

The most important feature of this function, known in the literature as Minty (or dual) gap function, is its convexity. However, it is in general nondifferentiable; subdifferentiability and related properties have been analysed in $[60,62,74,97,100]$. Furthermore, it can be difficult to evaluate $p^{M}$ since the optimization problem in (17) is generally not convex.

A cutting plane method for minimizing $p^{M}$ has been proposed in [74]: at each iteration it solves a linear programming problem, provided that $C$ is a polyhedron, and it converges to a solution to (VI) if $F$ is strictly monotone. Later, this method has been combined with the Tikhonov regularization technique in order to deal with monotone VIs [15].

Following the scheme described before for (VI), it is possible to regularize the function $p^{M}$ exploiting a bifunction $G$ which satisfies conditions (13). In fact, the function

$$
p_{G}^{M}(x):=\sup _{y \in C}[\langle F(y), x-y\rangle-G(x, y)]
$$

is a gap function for (MVI) (see [63]). Moreover, if the optimization problem in (18) has a unique solution $y(x)$, then $p_{G}^{M}$ is continuously differentiable and its gradient is given by

$$
\nabla p_{G}^{M}(x)=F(y(x))-\nabla_{x} G(x, y(x)) .
$$

In parallel with the analysis developed for $(\mathrm{VI})$, a descent method for the function $p_{G}^{M}$ has been proposed in [63]. Given any starting point $x^{0} \in C$, any sequence $\left\{x^{k}\right\}$ generated by an exact line search algorithm with descent direction given by $y(x)-x$ converges to the unique solution to (VI), provided that $C$ is compact, $F$ is continuously differentiable, $\nabla F$ is positive definite on $C$ and

$$
\nabla_{x} G(x, y)+\nabla_{y} G(x, y)=0, \quad \forall x, y \in C .
$$


Observe that the latter condition is fulfilled, for instance, by

$$
G(x, y)=\frac{1}{2}\langle x-y, M(x-y)\rangle .
$$

This algorithm is stated in [64] employing an inexact linesearch rule and replacing the assumption that $\nabla F(x)$ is positive definite for $x \in C$ with the strong monotonicity of $F$ on $C$.

A different regularization of the gap function $p^{M}$ has been proposed in [97], where the following function is considered:

$$
p_{\beta}^{M}(x):=\sup _{y \in C}\left[\langle F(y), x-y\rangle+\beta\|x-y\|^{2}\right],
$$

where $\beta$ is a positive parameter. This function is convex and lower semicontinuous as the original $p^{M}$. It is continuously differentiable provided that $F$ is so and the supremum in (19) is attained in a unique point. Moreover, if $F$ is strongly monotone on $C$ with modulus $\mu$, and $\beta \in(0, \mu]$, it is a gap function for (VI) and provides an error bound, i.e.

$$
\left\|x-x^{*}\right\| \leq \sqrt{p_{\beta}^{M}(x) / \beta}, \quad \forall x \in C,
$$

where $x^{*}$ is the unique solution to (VI).

\subsubsection{Gap functions based on conjugate duality}

Given a convex function $f: \mathbb{R}^{n} \rightarrow \mathbb{R}$ and a concave function $g: \mathbb{R}^{n} \rightarrow \mathbb{R}$, we recall that

$$
f^{*}(y):=\sup _{x \in \mathbb{R}^{n}}[\langle y, x\rangle-f(x)], \quad g_{*}(y):=\inf _{x \in \mathbb{R}^{n}}[\langle y, x\rangle-g(x)]
$$

are the Fenchel conjugate, in convex and concave sense respectively, of $f$ and $g$ (see e.g. [87]). Moreover, the Fenchel dual of the problem

$$
\inf _{x \in \mathbb{R}^{n}}[f(x)-g(x)]
$$

is defined as

$$
\sup _{y \in \mathbb{R}^{n}}\left[g_{*}(y)-f^{*}(y)\right]
$$

Note that the Fenchel dual of the constrained problem

$$
\inf _{x \in C} f(x)
$$

can be obtained defining $g(x)=-\delta_{C}(x)$, where $\delta_{C}$ is the indicator ${ }^{4}$ function of the set $C$, so that

$$
\inf _{x \in C} f(x)=\inf _{x \in \mathbb{R}^{n}}\left[f(x)-\left(-\delta_{C}(x)\right)\right]
$$

and the associated Fenchel dual turns out to be

$$
\sup _{y \in \mathbb{R}^{n}}\left[-f^{*}(y)+\inf _{x \in C}\langle y, x\rangle\right]
$$

\footnotetext{
${ }^{4} \delta_{C}$ is defined as follows: $\delta_{C}(x)=0$ if $x \in C$ and $\delta_{C}(x)=+\infty$ otherwise.
} 
When the feasible set $C$ is explicitly defined by convex constraints as in (3), the value of the Auslender gap function $p$ at a given point $x$ coincides (see [1,53]) with the opposite of the optimal value of the Fenchel dual of the problem

$$
\inf _{y \in C}\langle F(x), y-x\rangle .
$$

Moreover, the opposite of the optimal value of the so called Lagrangian dual and the FenchelLagrange dual associated with $P(x)$ leads to define a further gap function that coincides with

$$
p^{L}(x):=\inf _{\lambda \geq 0} \sup _{y \in \mathbb{R}^{n}}[\langle F(x), x-y\rangle-\langle\lambda, g(y)\rangle],
$$

which has been proposed in [39].

Similarly, considering the opposite of the optimal values of the Lagrange and of the Fenchel dual associated with the problem

$$
\inf _{y \in C}\langle F(y), y-x\rangle,
$$

which is equivalent to the one which appears in the right-hand side of (17), the following gap functions for (MVI) are defined [1]:

$$
\begin{gathered}
p^{M L}(x):=\inf _{\lambda \geq 0} \sup _{y \in \mathbb{R}^{n}}[\langle F(y), x-y\rangle-\langle\lambda, g(y)\rangle], \\
p^{M F}(x):=\inf _{p \in \mathbb{R}^{n}}\left\{\sup _{y \in \mathbb{R}^{n}}[\langle F(y), x-y\rangle+\langle p, y\rangle]+\delta_{C}^{*}(-p)\right\},
\end{gathered}
$$

where $\delta_{C}^{*}(x)=\sup _{y \in C}\langle x, y\rangle$ is the support function to the set $C$.

The proposed gap functions are all convex if $F$ is an affine monotone map.

\subsection{Unconstrained optimization reformulations}

In this section, we show merit functions which allow to reformulate (VI) as an unconstrained optimization problem.

\subsubsection{D-gap functions}

The difference of two regularized gap functions

$$
p_{\alpha \beta}(x):=p_{\alpha}(x)-p_{\beta}(x),
$$

where $p_{\alpha}$ and $p_{\beta}$ are defined by (14) with $0<\alpha<\beta$, is called D-gap function (where D stands for "difference"). This function is nonnegative on the whole space $\mathbb{R}^{n}$ and $p_{\alpha \beta}\left(x^{*}\right)=0$ if and only if $x^{*}$ is a solution to (VI) [98]. Therefore, solving (VI) is equivalent to finding the optimal solutions of the problem

$$
\min _{x \in \mathbb{R}^{n}} p_{\alpha \beta}(x) .
$$

When (VI) is a nonlinear complementarity problem, the D-gap function with $\beta=1 / \alpha$ and $\alpha \in(0,1)$ coincides with the implicit Lagrangian proposed and studied in [57, 81, 83]. 
Clearly, the D-gap function inherits the differentiability properties of $p_{\alpha}$ and $p_{\beta}$, i.e., if $F$ is differentiable, the function $p_{\alpha \beta}$ is also differentiable and

$$
\nabla p_{\alpha \beta}(x)=(\nabla F(x))^{T}\left[y_{\beta}(x)-y_{\alpha}(x)\right]+\beta \nabla_{x} G\left(x, y_{\beta}(x)\right)-\alpha \nabla_{x} G\left(x, y_{\alpha}(x)\right)
$$

where $y_{\alpha}(x)$ and $y_{\beta}(x)$ are the solutions of the optimization problem (14) with $\alpha$ and $\beta$ respectively. When the mapping $F$ is locally Lipschitz continuous, the D-gap function is also locally Lipschitz and the Clarke generalized gradient of $p_{\alpha \beta}$ satisfies a formula similar to (26) (see [72]).

The D-gap function is not convex in general and the stationary points of (25) may not be global minima. However, if $x^{*}$ is a stationary point, i.e. $\nabla p_{\alpha \beta}\left(x^{*}\right)=0$, and the Jacobian matrix $\nabla F\left(x^{*}\right)$ is positive definite, then $x^{*}$ is a solution to (VI) [98]. Notice that the positive definiteness of $\nabla F\left(x^{*}\right)$ can not be replaced by the strict monotonicity assumption on $F$ (see the counterexample in [98]). When the feasible set $C$ is a box, it is sufficient to assume that $\nabla F\left(x^{*}\right)$ is a $P$-matrix (i.e. its principal minors are all positive) to obtain the same conclusion [46]. In the special case of strongly monotone affine VIs, the D-gap function is convex (strongly convex) provided that the parameters $\alpha$ and $\beta$ are chosen so that the matrix

$$
A+A^{T}-\alpha I-\beta^{-1} A^{T} A
$$

is positive semidefinite (positive definite) [84].

Since the D-gap functions allow to reformulate (VI) as an unconstrained problem, the boundedness of their level sets is an important issue in order to develop minimization algorithms for solving problem (25). The level sets of the D-gap function, denoted by

$$
L(c):=\left\{x \in \mathbb{R}^{n}: p_{\alpha \beta}(x) \leq c\right\},
$$

are bounded for all $c \geq 0$ if either $C$ is bounded [46] or $F$ is strongly monotone $[84,85]$. Recently, it has been proved that monotonicity assumptions on $F$ are not needed for the boundedness of the level sets: in fact, the sets $L(c)$ are bounded for all $c \geq 0$ provided a coercivity condition stronger than (2) holds [56].

If $F$ is strongly monotone on $\mathbb{R}^{n}$ and either $F$ is Lipschitz continuous on $\mathbb{R}^{n}$ or $C$ is bounded, then $\sqrt{p_{\alpha \beta}}$ provides an error bound for $(\mathrm{VI})$, i.e. there exists a constant $M>0$ such that

$$
\left\|x-x^{*}\right\| \leq M \sqrt{p_{\alpha \beta}(x)}, \quad \forall x \in \mathbb{R}^{n},
$$

(see [98]). Notice that when $C$ is unbounded, the strong monotonicity on $F$, without the Lipschitz continuity assumption, is not sufficient to guarantee the same result (see the counterexample in [45]). However, when $F$ is strongly monotone, (VI) has a unique solution and hence it is possible to reformulate the problem by replacing the set $C$ by its intersection with a sphere large enough to contain the solution. In the special case where $C$ is a box, the strong monotonicity of $F$ can be replaced by the assumption that $F$ is a uniform $P$-function [46]. Recently, new global error bounds have been proposed in [55].

On the other hand, the strong monotonicity on $F$ only guarantees a local error bound on the level sets of the D-gap function [85], that is for any $c \geq 0$ there exists $M>0$ such that

$$
\left\|x-x^{*}\right\| \leq M \sqrt{p_{\alpha \beta}(x)}, \quad \forall x \in L(c) .
$$


Recently, this result has been extended to locally $\xi$-monotone and coercive mappings [55] and to general nonmonotone mappings [56].

There are several solution methods for VIs based on the minimization of D-gap functions. A descent method with Armijo-type line search has been proposed in [98]: at each iteration it exploits the search direction $d=r(x)+\rho s(x)$, where $r(x)=y_{\alpha}(x)-y_{\beta}(x), s(x)=$ $\alpha \nabla_{x} G\left(x, y_{\alpha}(x)\right)-\beta \nabla_{x} G\left(x, y_{\beta}(x)\right)$ and $\rho>0$ is a sufficiently small constant. This method converges to the solution to (VI) if $F$ is strongly monotone on $\mathbb{R}^{n}$ and either $F$ is Lipschitz continuous on $\mathbb{R}^{n}$ or $C$ is bounded. Another descent method was developed in [89] for solving monotone VIs with bounded feasible set. It is similar to the method proposed in [103] based on the Fukushima's regularized gap function: at each iteration it uses $d=y_{\alpha}(x)-y_{\beta}(x)$ as search direction along with a suitable update of the parameters $\alpha$ and $\beta$. A descent method for solving nonmonotone VIs, which is based on the minimization of the function $\sqrt{p_{\alpha \beta}}$, has been presented recently in [56].

A hybrid Newton method has been proposed in [84]: at each iteration, it finds the solution $z(x)$ of the linearized VI (10) at $x$ and it tries to use the direction $d=z(x)-x$ whenever it provides a sufficient decrease in the $\mathrm{D}$-gap function $p_{\alpha \beta}$; otherwise the direction $d=-\nabla p_{\alpha \beta}(x)$ is used. Then, an inexact line search is performed to get the next iterate. The generated sequence converges superlinearly to the unique solution $x^{*}$ to (VI) if $F$ is continuously differentiable and strongly monotone on $\mathbb{R}^{n}$. Furthermore, the convergence is quadratic if $\nabla F$ is Lipschitz continuous around $x^{*}$. A variant of this method has been proposed in [82] for box constrained VIs.

In [46] a nonsmooth Gauss-Newton type method for solving box constrained VIs has been presented. At each iteration, it solves a linear system of equations involving the generalized Hessian of the D-gap function and tries to use this vector as search direction if a descent condition is satisfied; otherwise the direction $d=-\nabla p_{\alpha \beta}(x)$ is used. Then an inexact line search is performed. The algorithm is globally and superlinearly convergent under suitable assumptions. A similar Gauss-Newton strategy has also been adopted in a trust region method for minimizing the D-gap function [90].

Another Newton type method for the solution of box constrained VIs is based on the reformulation of (VI) as a system of nonsmooth and nonlinear equations involving the natural residual. This method, based on the minimization of D-gap function, is globally and superlinearly convergent [47].

\subsubsection{Merit functions via the Moreau-Yosida regularization}

Another approach to get unconstrained optimization reformulations of (VI) is based on the Moreau-Yosida regularization of some gap functions [97].

The function

$$
p_{\alpha \lambda}^{M Y}(x):=\inf _{z \in C}\left\{\sup _{y \in C}\left[\langle F(z), z-y\rangle-\alpha\|y-z\|^{2}\right]+\lambda\|z-x\|^{2}\right\},
$$

with $\alpha \geq 0$ and $\lambda>0$, is derived from the Moreau-Yosida regularization of the regularized gap function $p^{F}$, with $M=\alpha I$. It is nonnegative on the whole space $\mathbb{R}^{n}$ and $p_{\alpha \lambda}^{M Y}\left(x^{*}\right)=0$ if and only if $x^{*}$ solves (VI).

Notice that this merit function may not be easy to evaluate in practice unless (VI) has a certain special structure (e.g. $F$ is affine and $C$ is a polyhedron [97]). However, it enjoys some 
nice theoretical properties that other merit functions do not have. For instance, if the minimum problem in (28) has a unique solution $z_{\alpha \lambda}(x)$ for each $x \in \mathbb{R}^{n}$, then $p_{\alpha \lambda}^{M Y}$ is differentiable on $\mathbb{R}^{n}$ and

$$
\nabla p_{\alpha \lambda}^{M Y}(x)=2 \lambda\left[x-z_{\alpha \lambda}(x)\right]
$$

even if $F$ is not differentiable.

In general, the function $p_{\alpha \lambda}^{M Y}$ is not convex. However, if the gap function $p$ is convex, then $p_{0 \lambda}^{M Y}$ is differentiable and convex on $\mathbb{R}^{n}$ for any $\lambda>0$; while if the regularized gap function $p^{F}$, with $M=\alpha I$, is convex then $p_{\alpha \lambda}^{M Y}$ is differentiable and convex on $\mathbb{R}^{n}$ for any $\lambda>0$.

The function $p_{\alpha \lambda}^{M Y}$ provides also a global error bound under the strong monotonicity of $F$ (without assuming Lipschitz continuity as is the case for the D-gap functions). In fact, if $F$ is strongly monotone on $C$ with modulus $\mu, \alpha \in[0, \mu)$ and $\lambda>0$, then

$$
\frac{1}{2} \min \{\mu-\alpha, \lambda\}\left\|x-x^{*}\right\|^{2} \leq p_{\alpha \lambda}^{M Y}(x) \leq \lambda\left\|x-x^{*}\right\|^{2}, \quad \forall x \in \mathbb{R}^{n},
$$

i.e. the growth rate of $p_{\alpha \lambda}^{M Y}$ is in the order of the squared distance from the unique solution $x^{*}$ to (VI).

When $F$ satisfies suitable monotonicity assumptions, further merit functions for (VI) can be obtained by the Moreau-Yosida regularization of Minty gap functions. The function

$$
p_{\beta \lambda}^{M}(x):=\inf _{z \in C}\left\{\sup _{y \in C}\left[\langle F(y), z-y\rangle+\beta\|y-z\|^{2}\right]+\lambda\|z-x\|^{2}\right\},
$$

with $\beta \geq 0$ and $\lambda>0$, is the Moreau-Yosida regularization of Minty gap function $p^{M}$ (when $\beta=0$ ) and regularized Minty gap function $p_{\beta}^{M}$ (when $\beta>0$ ). If $F$ is pseudomonotone on $C$, then $p_{0 \lambda}^{M}$ turns out to be a merit function for (VI) for any $\lambda>0$, because it is nonnegative on $\mathbb{R}^{n}$ and $p_{0 \lambda}^{M}\left(x^{*}\right)=0$ if and only if $x^{*}$ solves (VI). Furthermore, if $F$ is strongly monotone with modulus $\mu$, then the same happens for $p_{\beta \lambda}^{M}$ provided that $\beta \in[0, \mu]$.

Note that also $p_{\beta \lambda}^{M}$ may not be easy to evaluate in practice, but some nice theoretical properties hold. In fact, it is differentiable and convex on $\mathbb{R}^{n}$ for any $\beta \geq 0$ and $\lambda>0$, without making any additional assumption on $F$. Moreover, if $F$ is strongly monotone on $C$ with modulus $\mu$, $\beta \in(0, \mu]$ and $\lambda>0$, then the quadratic growth rate of $p_{\beta \lambda}^{M}$ is ensured, i.e.

$$
\frac{1}{2} \min \{\beta, \lambda\}\left\|x-x^{*}\right\|^{2} \leq p_{\beta \lambda}^{M}(x) \leq \lambda\left\|x-x^{*}\right\|^{2}, \quad \forall x \in \mathbb{R}^{n},
$$

where $x^{*}$ is the unique solution to (VI).

\subsection{An application to traffic network equilibrium problems}

A traffic network consists of a set of nodes $\mathcal{N}$, a set of $\operatorname{arcs} \mathcal{A} \subseteq \mathcal{N} \times \mathcal{N}$ and a set of origin/destination pairs $\mathcal{W} \subseteq \mathcal{N} \times \mathcal{N}$. For each $\mathrm{O} / \mathrm{D}$ pair $w$, a traffic demand $d_{w}$ has to be distributed among the paths connecting $w$. We denote $\mathcal{P}_{w}$ the set of all paths connecting $w, x_{p}$ the flow on path $p$ and $x=\left(x_{p}\right)_{p \in \mathcal{P}_{w}, w \in \mathcal{W}}$ the vector of all path flows. The set of feasible path flows is given by

$$
X=\left\{x \geq 0: \quad \sum_{p \in \mathcal{P}_{w}} x_{p}=d_{w}, \quad \forall w \in \mathcal{W}\right\} .
$$


The flow $f_{a}$ on each $\operatorname{arc} a$ is the sum of all flows on paths to which the arc belongs, hence the arc flow vector $f=\left(f_{a}\right)_{a \in \mathcal{A}}$ can be written as $f=\Delta x$, where $\Delta$ is the arc-path incidence matrix:

$$
\Delta_{a, p}= \begin{cases}1 & \text { if } a \in p, \\ 0 & \text { otherwise }\end{cases}
$$

For each $\operatorname{arc} a$, there is a nonnegative cost function $t_{a}(f)$, which represents the travel time associated with $\operatorname{arc} a$ and depends on the arc flow vector $f$. The corresponding path cost function is assumed to be additive, i.e. the travel time $T_{p}(x)$ on path $p$ is the sum of the travel times of the arcs belonging to $p$ :

$$
T_{p}(x)=\sum_{a \in p} t_{a}(\Delta x) .
$$

According to the Wardrop equilibrium principle [95], a path flow $x^{*} \in X$ is called a network equilibrium if it is positive only on minimum cost paths, i.e. the following implication

$$
x_{p}^{*}>0 \quad \Longrightarrow \quad T_{p}\left(x^{*}\right)=\min _{q \in \mathcal{P}_{w}} T_{q}\left(x^{*}\right)
$$

holds for any $\mathrm{O} / \mathrm{D}$ pair $w \in \mathcal{W}$ and path $p \in \mathcal{P}_{w}$.

It is well-known [24] that the problem of finding network equilibria is equivalent to solving the following variational inequality:

$$
\text { find } x^{*} \in X \text { such that }\left\langle T\left(x^{*}\right), y-x^{*}\right\rangle \geq 0 \text {, for all } y \in X \text {. }
$$

Next example shows how the merit function approach for VIs can be applied to network equilibria.

Example 3.1. Consider the network in Fig. 1 with two $O / D$ pairs: $w_{1}=(1,4)$ with demand $d_{1}=4$ and $w_{2}=(1,5)$ with $d_{2}=6$. Each $O / D$ pair is connected by two paths: $\mathcal{P}_{w_{1}}=\{(1,2),(2,4) ;(1,3),(3,4)\}$ and $\mathcal{P}_{w_{2}}=\{(1,2),(2,5) ;(1,3),(3,5)\}$. We denote the flow on paths as $x_{1} \ldots, x_{4}$, respectively. Hence the set of feasible path flows is given by

$$
X=\left\{x \in \mathbb{R}_{+}^{4}: x_{1}+x_{2}=4, \quad x_{3}+x_{4}=6\right\} .
$$

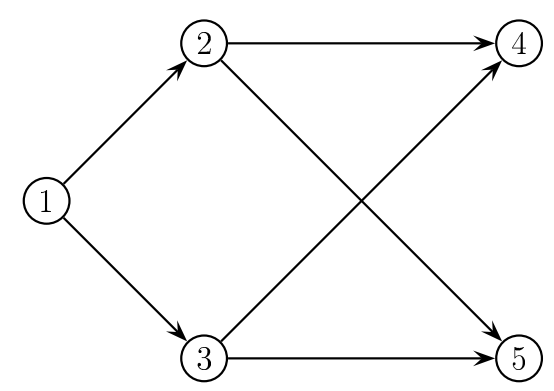

Figure 1: Traffic network in Example 3.1. 
Assume that the arc cost functions are defined as follows:

$$
\left\{\begin{aligned}
t_{12} & :=f_{12}+1=x_{1}+x_{3}+1 \\
t_{13} & :=3 f_{13}+2=3\left(x_{2}+x_{4}\right)+2 \\
t_{24} & :=2 f_{24}+f_{34}+1=2 x_{1}+x_{2}+1 \\
t_{25} & :=2 f_{25}+f_{35}+3=2 x_{3}+x_{4}+3 \\
t_{34} & :=f_{34}+2=x_{2}+2 \\
t_{35} & :=4 f_{35}+1=4 x_{4}+1
\end{aligned}\right.
$$

thus the corresponding path costs are

$$
\left\{\begin{array}{l}
T_{1}=t_{12}+t_{24}=3 x_{1}+x_{2}+x_{3}+2, \\
T_{2}=t_{13}+t_{34}=4 x_{2}+3 x_{4}+4 \\
T_{3}=t_{12}+t_{25}=x_{1}+3 x_{3}+x_{4}+4 \\
T_{4}=t_{13}+t_{35}=3 x_{2}+7 x_{4}+3
\end{array}\right.
$$

i.e. the operator of $V I(30)$ is $T(x)=A x+b$, with

$$
A=\left(\begin{array}{llll}
3 & 1 & 1 & 0 \\
0 & 4 & 0 & 3 \\
1 & 0 & 3 & 1 \\
0 & 3 & 0 & 7
\end{array}\right), \quad b=\left(\begin{array}{l}
2 \\
4 \\
4 \\
3
\end{array}\right) .
$$

Note that the matrix $A$ is positive definite, thus the mapping $T$ is strongly monotone and there exists a unique solution of VI (30), i.e. a unique network equilibrium.

We now consider the Fukushima's regularized gap function (7) with $M=I$, i.e.

$$
p^{F}(x)=\max _{y \in X}\left[\langle T(x), x-y\rangle-\frac{1}{2}\|x-y\|^{2}\right] .
$$

This function is continuously differentiable and strongly convex since the matrix $A+A^{T}-I$ is positive definite. In Fig. 2 we show the graph of $p^{F}$ defined on the 2-dimensional space $\left(x_{1}, x_{3}\right)$, with $x_{1} \in[0,4]$ and $x_{3} \in[0,6]$ (the demand constraints allow to express variables $x_{2}$ and $x_{4}$ as function of $x_{1}$ and $x_{3}$ ).

The descent algorithm (8)-(9) applied to $p^{F}$ can be exploited to compute the network equilibrium. Table 1 reports the first four iterations of the algorithm (implemented in MATLAB) starting from the feasible flow $(4,0,6,0)$.

\begin{tabular}{c|rrrr|c}
\hline Iteration & $x_{1}$ & $x_{2}$ & $x_{3}$ & $x_{4}$ & $p^{F}(x)$ \\
\hline 0 & 4.000000 & 0 & 6.000000 & 0 & $1.5000 \mathrm{e}+02$ \\
1 & 2.679622 & 1.320378 & 4.019434 & 1.980566 & $8.8840 \mathrm{e}-03$ \\
2 & 2.631471 & 1.368529 & 4.052452 & 1.947548 & $1.5178 \mathrm{e}-06$ \\
3 & 2.631587 & 1.368413 & 4.052626 & 1.947374 & $2.5929 \mathrm{e}-10$ \\
4 & 2.631579 & 1.368421 & 4.052632 & 1.947368 & $2.8747 \mathrm{e}-14$ \\
\hline
\end{tabular}

Table 1: Numerical results of the descent algorithm (8)-(9) applied to Example 3.1. 


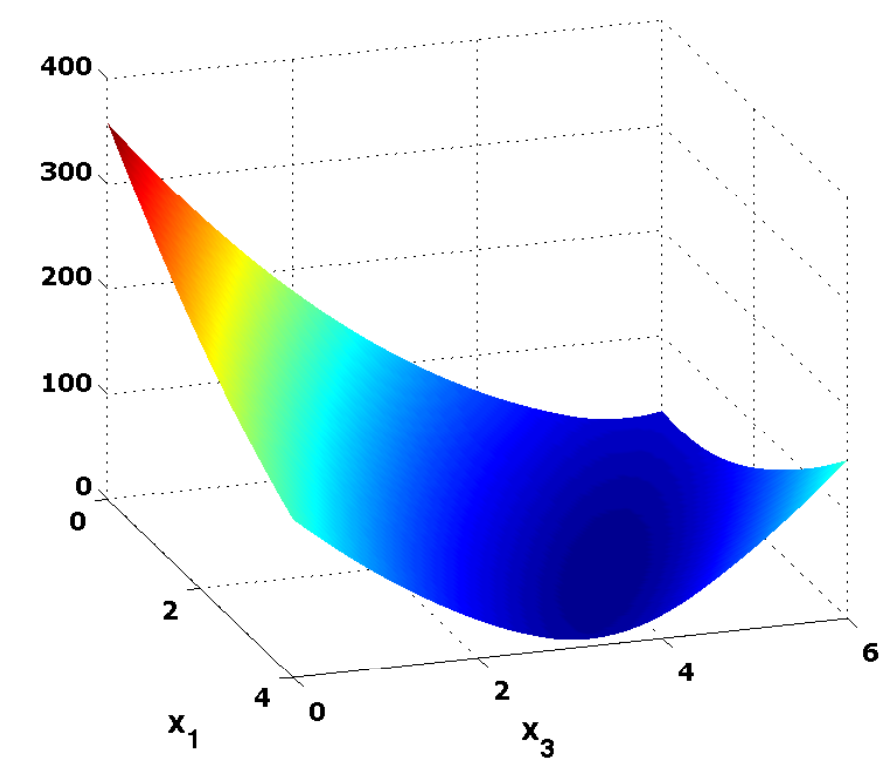

Figure 2: The regularized gap function $p^{F}$ with $M=I$ in Example 3.1.

Note that the path costs corresponding to the equilibrium solution

$$
x^{*}=(2.6316,1.3684,4.0526,1.9474)
$$

are

$$
T\left(x^{*}\right)=(15.3158,15.3158,20.7368,20.7368),
$$

i.e. the two paths connecting each $O / D$ pair have the same cost. Furthermore, the Lagrange multipliers $\lambda^{*}$ associated with $x^{*}$ in the KKT conditions (4) coincide with the equilibrium costs, i.e. $\lambda^{*}=(15.3158,20.7368)$.

Figure 3 shows the graph of the D-gap function $p_{\alpha \beta}$, with $\alpha=1, \beta=2$ and $G(x, y)=$ $\|x-y\|^{2} / 2$, defined on the space $\left(x_{1}, x_{3}\right)$. Note that this function is always nonnegative (even in unfeasible points) and its global minimum is $x^{*}$.

\section{Merit functions for quasi-variational inequalities}

In this section we consider the merit function approach for quasi-variational inequalities (QVIs), i.e. VIs in which the feasible region depends on the variable $x$. Given a vector-valued mapping $F: \mathbb{R}^{n} \rightarrow \mathbb{R}^{n}$ and a set-valued mapping $C: \mathbb{R}^{n} \rightrightarrows \mathbb{R}^{n}$, such that $C(x)$ are closed and convex sets for any $x \in \mathbb{R}^{n}$, the QVI is defined as follows:

$$
\text { find } x^{*} \in C\left(x^{*}\right) \text { such that }\left\langle F\left(x^{*}\right), y-x^{*}\right\rangle \geq 0 \text {, for all } y \in C\left(x^{*}\right) \text {. }
$$




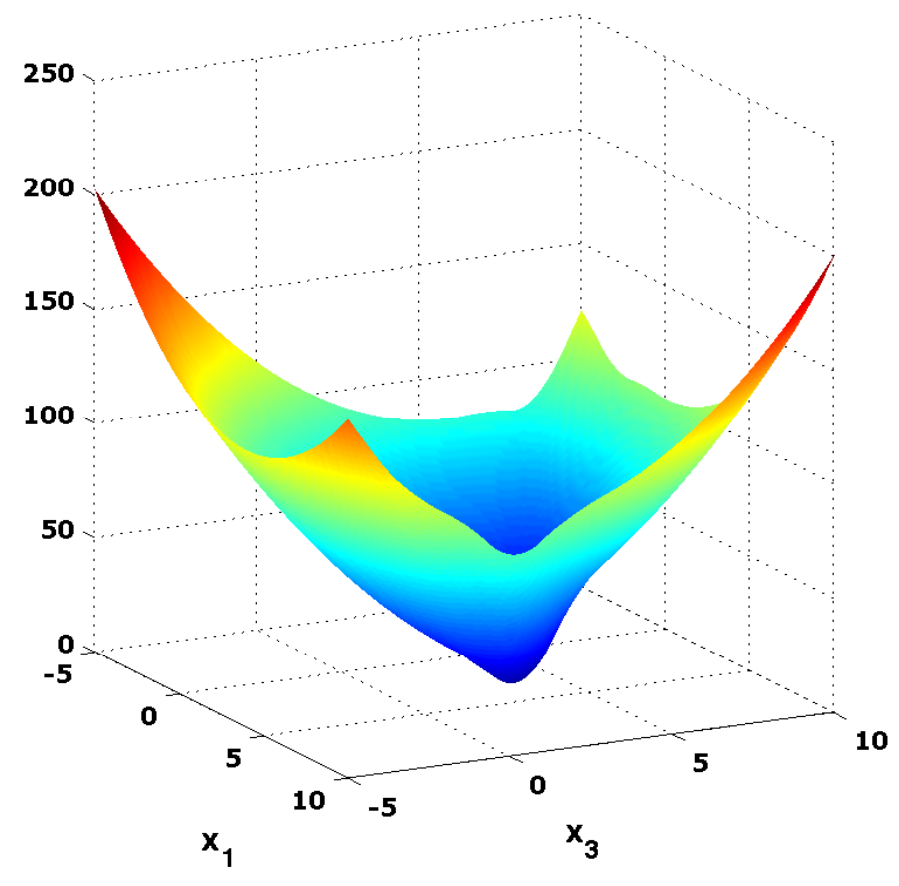

Figure 3: The D-gap function $p_{\alpha \beta}$ with $\alpha=1, \beta=2$ and $G(x, y)=\|x-y\|^{2} / 2$ in Example 3.1. 
When all the sets $C(x)$ coincide with the same set $C$, (QVI) collapses to (VI). The set of fixed points of the mapping $C$, i.e.

$$
X:=\left\{x \in \mathbb{R}^{n}: x \in C(x)\right\},
$$

is the feasible region of (QVI). In the following we suppose that sets $C(x)$ are defined by constraints, i.e.,

$$
C(x):=\left\{y \in \mathbb{R}^{n}: g_{i}(x, y) \leq 0, \quad i=1, \ldots, m\right\},
$$

where the functions $g_{i}: \mathbb{R}^{n} \times \mathbb{R}^{n} \rightarrow \mathbb{R}$ are assumed to be continuous and $g_{i}(x, \cdot)$ convex for any fixed $x \in \mathbb{R}^{n}$. Furthermore, in order to guarantee the convexity of the set $X$, we assume that the functions $x \mapsto g_{i}(x, x)$ are convex for all $i=1, \ldots, m$.

QVIs were introduced in $[11,12]$ and subsequently exploited to model several finite and infinite-dimensional problems (see [10, 22, 31, 32] and references therein).

Some merit functions have been proposed in the literature extending to QVIs similar ideas developed for VIs. Similarly to VIs, the reformulation of (QVI) as a fixed point problem leads to define a merit function. In fact, it follows from the definition that $x$ solves (QVI) if and only if $x=\pi_{C(x)}(x-F(x))$, thus $\left\|x-\pi_{C(x)}(x-F(x))\right\|$ is a merit function for (QVI). Another approach is based on reformulating (QVI), under suitable constraint qualifications, as a complementarity problem via the following KKT conditions:

$$
\begin{cases}F(x)+\sum_{i=1}^{m} \lambda_{i} \nabla_{y} g_{i}(x, x)=0, & \\ \lambda_{i} g_{i}(x, x)=0, & i=1, \ldots, m, \\ \lambda_{i} \geq 0, \quad g_{i}(x, x) \leq 0, & i=1, \ldots, m .\end{cases}
$$

Recently, a solution method based on these conditions has been proposed in [31].

A straightforward extension of the gap function (5) to QVIs is defined as follows [39]:

$$
\mathrm{p}(x):=\sup _{y \in C(x)}\langle F(x), x-y\rangle .
$$

This function is nonnegative on the set $X$ and $x^{*}$ solves (QVI) if and only if $x^{*} \in X$ and $\mathrm{p}\left(x^{*}\right)=0$. However, the gap function $\mathrm{p}$ is nondifferentiable and it may occur that $\mathrm{p}(x)=+\infty$ for some point of $X$.

The regularized gap function (7) has been extended to QVIs in [38] and is defined by

$$
\mathrm{p}_{\alpha}(x):=\max _{y \in C(x)}\left[\langle F(x), x-y\rangle-\frac{\alpha}{2}\|x-y\|^{2}\right] .
$$

This function is a gap function, it is finite and the maximum in (32) is attained in a unique point $y_{\alpha}(x)$, provided that the set $C(x)$ is nonempty. Actually, it is possible to define $\mathrm{p}_{\alpha}$ replacing the regularization term $\alpha\|x-y\|^{2} / 2$ and the set $C(x)$ with more general expressions satisfying suitable conditions $[38,91]$.

In contrast to VIs, this function is nondifferentiable even if $F$ is so (see examples in [43]). If $F$ and $g_{i}$ are continuously differentiable and a constraint qualification holds, then $\mathrm{p}_{\alpha}$ is directionally differentiable everywhere and its directional derivative at $x$ along direction $d$ is given by

$$
\mathrm{p}_{\alpha}^{\prime}(x ; d)=\min _{\lambda \in \Lambda_{\alpha}(x)}\left\langle F(x)-\left[(\nabla F(x))^{T}-\alpha I\right]\left[y_{\alpha}(x)-x\right]-\sum_{i=1}^{m} \lambda_{i} \nabla_{x} g_{i}\left(x, y_{\alpha}(x)\right), d\right\rangle,
$$


where $\Lambda_{\alpha}(x)$ is the set of Lagrange multipliers associated with $y_{\alpha}(x)$, i.e.,

$$
\begin{aligned}
\Lambda_{\alpha}(x)=\left\{\lambda \in \mathbb{R}_{+}^{m}: \quad\right. & F(x)+\alpha\left[y_{\alpha}(x)-x\right]+\sum_{i=1}^{m} \lambda_{i} \nabla_{y} g_{i}\left(x, y_{\alpha}(x)\right)=0, \\
& \left.\lambda_{i} g_{i}\left(x, y_{\alpha}(x)\right)=0, \quad i=1, \ldots, m\right\},
\end{aligned}
$$

(see [38]). Furthermore, $\mathrm{p}_{\alpha}$ turns out to be continuously differentiable in the special case of QVI with 'moving sets', i.e. when $C(x)=Q+c(x)$, where $Q$ is a closed and convex set and $c: \mathbb{R}^{n} \rightarrow \mathbb{R}^{n}$, provided that mappings $F$ and $c$ are continuously differentiable [27]. Recently, this latter result has been extended to QVIs with generalized moving sets [43].

Similarly to VIs, the regularized gap function $\mathrm{p}_{\alpha}$ is nonconvex in general. In [91] it is proved that, whenever $\mathrm{p}_{\alpha}$ is directionally differentiable, a stationary point $x^{*}$ of $\mathrm{p}_{\alpha}$ on $X$, i.e.

$$
\mathrm{p}_{\alpha}^{\prime}\left(x^{*} ; y-x^{*}\right) \geq 0, \quad \forall y \in X,
$$

is a solution to (QVI) provided that the matrix $\nabla F\left(x^{*}\right)$ is positive definite and

$$
\lambda_{i}\left\langle\nabla_{x} g_{i}\left(x^{*}, y_{\alpha}\left(x^{*}\right)\right), y_{\alpha}\left(x^{*}\right)-x^{*}\right\rangle \geq 0
$$

for all $i=1, \ldots, m$ and $\lambda \in \Lambda(x)$. Notice that in [91] the key assumption $y_{\alpha}\left(x^{*}\right) \in X$ is not explicitly stated in the statement, but it is exploited in the proof and must be therefore considered as hypothesis.

An unconstrained minimization reformulation of (QVI) can be obtained via the D-gap functions, i.e. the difference of two regularized gap functions. In fact, given $0<\alpha<\beta$, the function

$$
\mathrm{p}_{\alpha \beta}(x):=\mathrm{p}_{\alpha}(x)-\mathrm{p}_{\beta}(x)
$$

is nonnegative on $\mathbb{R}^{n}$ and $\mathrm{p}_{\alpha \beta}\left(x^{*}\right)=0$ if and only if $x^{*}$ solves (QVI). The directional differentiability of $\mathrm{p}_{\alpha \beta}$ directly follows from that of $\mathrm{p}_{\alpha}$ and $\mathrm{p}_{\beta}$.

The functions $\sqrt{\mathrm{p}_{\alpha}}$ and $\sqrt{\mathrm{p}_{\alpha \beta}}$ provide error bound results for (QVI) provided that $F$ is strongly monotone and Lipschitz continuous on $\mathbb{R}^{n}$ and an additional technical assumption on the Euclidean projection on the sets $C(x)$ is fulfilled [41]. Another error bound result based on the function $\sqrt{p_{\alpha}}$ has been recently proved in [9].

\subsection{An application to generalized Nash equilibrium problems}

Let us consider a noncooperative game with $N$ players, in which each player $i$ controls a set of variables $x_{i} \in \mathbb{R}^{n_{i}}$. The vector of all players strategies is denoted by $x=\left(x_{1}, \ldots, x_{N}\right) \in \mathbb{R}^{n}$, with $n=n_{1}+\ldots, n_{N}$; the vector $x$ is also denoted by $x=\left(x_{i}, x_{-i}\right)$, where $x_{-i}$ denotes the strategy vector of all the players different from player $i$. Each player $i$ has a cost function $\theta_{i}: \mathbb{R}^{n} \rightarrow \mathbb{R}$, which possibly depends on all players strategies $x$, and a feasible set $X_{i}\left(x_{-i}\right) \subseteq \mathbb{R}^{n_{i}}$, possibly depending on the rival players' strategies $x_{-i}$.

A generalized Nash equilibrium (GNE) of the game is a vector

$$
x^{*}=\left(x_{1}^{*}, \ldots, x_{N}^{*}\right) \in X_{1}\left(x_{-1}^{*}\right) \times \cdots \times X_{N}\left(x_{-N}^{*}\right)
$$

such that, for any $i=1, \ldots, N, x_{i}^{*}$ is an optimal solution of the following optimization problem:

$$
\min _{x_{i}} \theta_{i}\left(x_{i}, x_{-i}^{*}\right) \quad \text { subject to } \quad x_{i} \in X_{i}\left(x_{-i}^{*}\right) .
$$


In other words, $x^{*}$ is a GNE if no player can improve its own cost function by unilaterally changing its strategy.

It is well-known (see e.g. [30]) that under the following assumptions:

- $\theta_{i}$ is continuously differentiable for any $i=1, \ldots, N$,

- $\theta_{i}\left(\cdot, x_{-i}\right)$ is convex for any $x_{-i}$ and $i=1, \ldots, N$,

- the feasible sets $X_{i}\left(x_{-i}\right)$ are closed and convex for all $x \in \mathbb{R}^{n}$ and $i=1, \ldots, N$,

the problem of finding GNE is equivalent to solving the QVI with operator

$$
F(x)=\left(\nabla_{x_{1}} \theta_{1}(x), \ldots, \nabla_{x_{N}} \theta_{N}(x)\right)
$$

and set-valued mapping

$$
C(x)=X_{1}\left(x_{-1}\right) \times \ldots X_{N}\left(x_{-N}\right) .
$$

Example 4.1. (see [20]) Consider a two-person noncooperative game, in which player $i$ select the coordinate $x_{i} \in \mathbb{R}$ subject to a individual constraint $x_{i} \leq 0$ and a shared constraint $x_{1}+x_{2} \leq-1$. The aim of player $i$ is to minimize the (squared) distance between $\left(x_{1}, x_{2}\right)$ and his favourite goal $P_{i} \in \mathbb{R}^{2}$, with $P_{1}=(1,0)$ and $P_{2}=(0,1)$. Thus the optimization problems of the two players are defined as follows:

$$
\text { Player 1: }\left\{\begin{array} { l } 
{ \operatorname { m i n } _ { x _ { 1 } } ( x _ { 1 } - 1 ) ^ { 2 } + x _ { 2 } ^ { 2 } } \\
{ x _ { 1 } \leq 0 } \\
{ x _ { 1 } + x _ { 2 } \leq - 1 }
\end{array} \quad \text { Player 2: } \left\{\begin{array}{l}
\min _{x_{2}} x_{1}^{2}+\left(x_{2}-1\right)^{2} \\
x_{2} \leq 0 \\
x_{1}+x_{2} \leq-1
\end{array}\right.\right.
$$

The set of GNE of the game coincide with the solution set of the QVI given by $F(x)=\left(2 x_{1}-\right.$ $\left.2,2 x_{2}-2\right)$ and

$$
C(x)=\left(-\infty, \min \left\{0,-1-x_{2}\right\}\right] \times\left(-\infty, \min \left\{0,-1-x_{1}\right\}\right] .
$$

The feasible region of the QVI, i.e. the set of fixed point of the set-valued mapping $C$, is

$$
X=\left\{x \in \mathbb{R}^{2}: x_{1} \leq 0, x_{2} \leq 0, x_{1}+x_{2} \leq-1\right\} .
$$

It is easy to check that the solution set of the QVI is the segment connecting $(-1,0)$ and $(0,-1)$.

The value of the gap function (31) can be explicitly computed:

$$
\begin{aligned}
& \mathrm{p}(x)=\sup _{y \in C(x)}\langle F(x), x-y\rangle \\
& =\sup _{y \in C(x)}\left[2\left(x_{1}-1\right)\left(x_{1}-y_{1}\right)+2\left(x_{2}-1\right)\left(x_{2}-y_{2}\right)\right] \\
& =2 x_{1}\left(x_{1}-1\right)+2 x_{2}\left(x_{2}-1\right)+\sup _{y_{1} \leq \min \left\{0,-1-x_{2}\right\}} 2\left(1-x_{1}\right) y_{1} \\
& +\sup _{y_{2} \leq \min \left\{0,-1-x_{1}\right\}} 2\left(1-x_{2}\right) y_{2} \\
& =\left\{\begin{aligned}
2 x_{1}\left(x_{1}-1\right)+2 x_{2}\left(x_{2}-1\right)+ & \\
\quad+2\left(1-x_{1}\right) \min \left\{0,-1-x_{2}\right\}+ & \\
+2\left(1-x_{2}\right) \min \left\{0,-1-x_{1}\right\}, & \text { if } x_{1} \leq 1 \text { and } x_{2} \leq 1, \\
+\infty, & \text { otherwise. }
\end{aligned}\right.
\end{aligned}
$$


This function is equal to zero on the solution set, but is not finite everywhere on $\mathbb{R}^{2}$ and it is not differentiable on the half-lines $\{-1\} \times(-\infty, 1]$ and $(-\infty, 1] \times\{-1\}$.

Figures 4 and 5 show the graphs of the regularized gap function $\mathrm{p}_{\alpha}$, with $\alpha=5$, and the D-gap function $\mathrm{p}_{\alpha \beta}$, with $\alpha=5$ and $\beta=10$, respectively. Note that both functions are finite on $\mathbb{R}^{2}$ and equal to zero in the solution set; $\mathrm{p}_{\alpha}$ is negative in points not belonging to $X$, while $\mathrm{p}_{\alpha \beta}$ is nonnegative on the whole space $\mathbb{R}^{2}$.

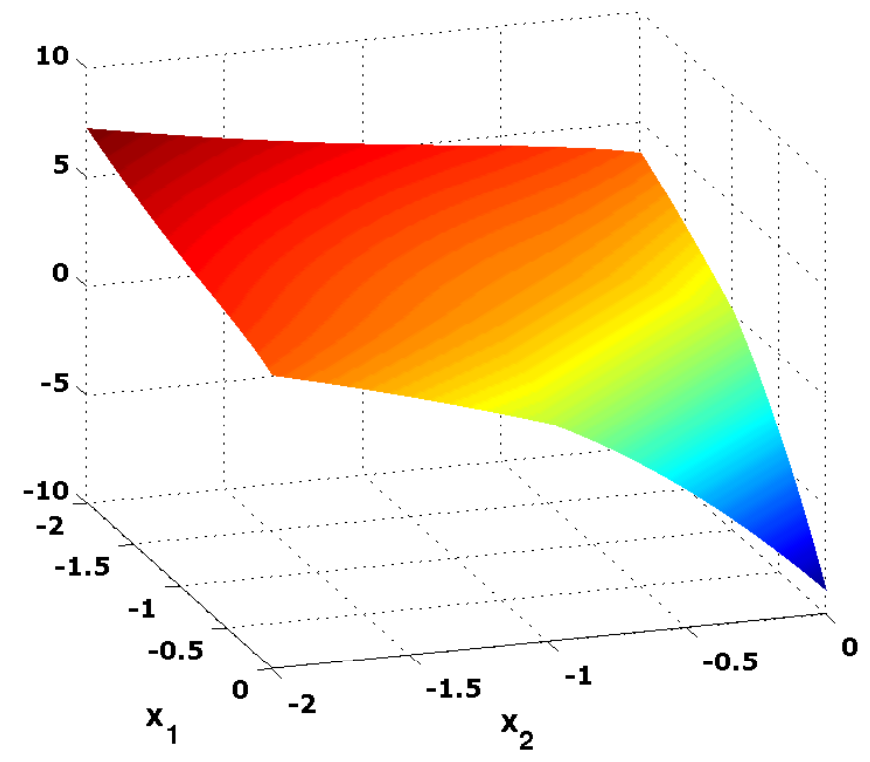

Figure 4: The regularized gap function $\mathrm{p}_{\alpha}$ with $\alpha=5$ in Example 4.1.

\section{Merit functions for abstract equilibrium problems}

The abstract equilibrium problem is a general mathematical model which includes optimization, multiobjective optimization, variational inequalities, fixed point and complementarity problems, Nash equilibria in noncooperative games and inverse optimization as special cases (see [14, 19]). It is defined as follows:

$$
\text { find } x^{*} \in C \text { such that } f\left(x^{*}, y\right) \geq 0 \text {, for all } y \in C \text {, }
$$

where $C$ is a closed and convex subset of $\mathbb{R}^{n}$ and $f: \mathbb{R}^{n} \times \mathbb{R}^{n} \rightarrow \mathbb{R}$ is a bifunction such that $f(x, \cdot)$ is convex and satisfies $f(x, x)=0$ for all $x \in C$. Setting $f(x, y)=\langle F(x), y-x\rangle$ we obtain $(\mathrm{VI})$.

In the last decade several merit functions for (EP) have been introduced in the literature. These functions often extend to (EP) those originally conceived for VIs. For instance, a direct 


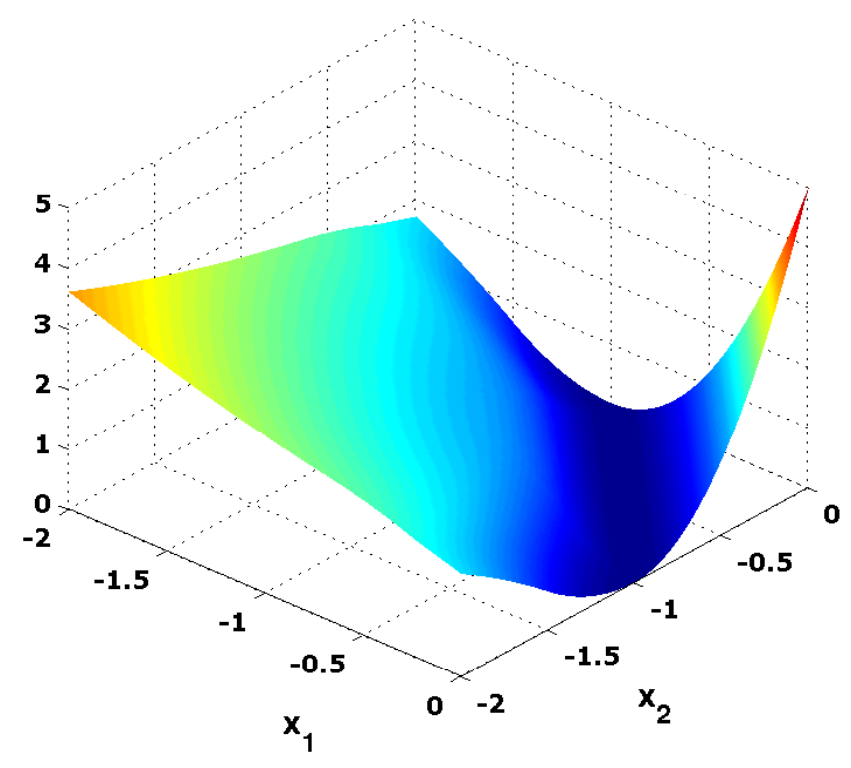

Figure 5: The D-gap function $\mathrm{p}_{\alpha \beta}$ with $\alpha=5$ and $\beta=10$ in Example 4.1.

extension of gap function (5) from VIs to (EP) is defined as follows [65]:

$$
\mathbf{p}(x):=\sup _{y \in C}[-f(x, y)] .
$$

This function is nonnegative on $C$ and $x^{*}$ solves (EP) if and only if $x^{*} \in C$ and $\mathbf{p}\left(x^{*}\right)=0$. However, $\mathbf{p}$ has the same disadvantages of function (5), i.e. it is in general neither finite, nor differentiable nor convex. For these reasons, the regularized gap function has been proposed [65]:

$$
\mathbf{p}_{\alpha}(x):=\max _{y \in C}\left[-f(x, y)-\frac{\alpha}{2}\|x-y\|^{2}\right] .
$$

It allows to reformulate (EP) as the problem of minimizing $\mathbf{p}_{\alpha}$ on $C$, it is continuously differentiable, if the bifunction $f$ is so, and

$$
\nabla \mathbf{p}_{\alpha}(x)=-\nabla_{x} f\left(x, y_{\alpha}(x)\right)-\alpha\left[x-y_{\alpha}(x)\right],
$$

where $y_{\alpha}(x)$ is the unique maximizer of problem (33). Note that the regularization term $\|y-x\|^{2}$ can be replaced by a more general bifunction $G$ satisfying condition (13) (see [65]). Similarly to VIs, the regularized gap function is nonconvex in general. However, if $x^{*}$ is a stationary point of $\mathbf{p}_{\alpha}$ on $C$, i.e.,

$$
\left\langle\nabla \mathbf{p}_{\alpha}\left(x^{*}\right), y-x^{*}\right\rangle \geq 0, \quad \forall y \in C,
$$

and $f$ is strictly $\nabla$-monotone on $C$, i.e.,

$$
\left\langle\nabla_{x} f(x, y)+\nabla_{y} f(x, y), y-x\right\rangle>0, \quad \forall x, y \in C \text { with } x \neq y,
$$


then $x^{*}$ is a solution to (EP). The strict $\nabla$-monotonicity of $f$ plays a role similar to those of positive definiteness of $\nabla F$ for VIs. In fact, it guarantees, in addition to the above "stationarity" property, that $y_{\alpha}(x)-x$ is a descent direction for $\mathbf{p}_{\alpha}$ at any non-stationary point $x$. Solution methods based on the minimization of $\mathbf{p}_{\alpha}$ along this direction have been developed in [21, 65]. An inexact version of these methods has been proposed in [26].

A descent method which does not require the strict $\nabla$-monotonicity of $f$ has been introduced in [13]. It is similar to that developed in [103] for VIs: at any iteration it performs a line search if $y_{\alpha}(x)-x$ is a descent direction for $\mathbf{p}_{\alpha}$ at $x$, otherwise the value of $\alpha$ is reduced. Convergence is guaranteed provided that $C$ is bounded and $f$ satisfies the following condition:

$$
f(x, y)+\left\langle\nabla_{x} f(x, y), y-x\right\rangle \geq 0, \quad \forall x, y \in C .
$$

The latter condition is neither stronger nor weaker than strict $\nabla$-monotonicity and it is satisfied if $f(\cdot, y)$ is concave for all $y \in C$ (see [13]).

Similarly to VIs, function $\sqrt{\mathbf{p}_{\alpha}}$ provides error bound results under suitable monotonicity assumptions on $f$ (see $[21,51,65]$ ).

Since the evaluation of the regularized gap function $\mathbf{p}_{\alpha}$ could be computationally expensive if $C$ is defined as in (3) by nonlinear constraints, a variant of function $\mathbf{p}_{\alpha}$ can be exploited as in the case of VIs. In [16] the following function has been introduced:

$$
\mathbf{p}_{\alpha}^{\mathrm{BP}}(x):=\max _{y \in T(x)}\left[-f(x, y)-\frac{\alpha}{2}\|x-y\|^{2}\right],
$$

where $T(x)$ is the outer polyhedral approximation of $C$ at $x$ defined as in (12). This function turns out to be a locally Lipschitz gap function for (EP). Furthermore, if $g_{i}$ 's are continuously differentiable and a constraint qualification holds, then $\mathbf{p}_{\alpha}^{\mathbf{B P}}$ is directionally differentiable. Solution methods for (EP) exploiting this merit function have been proposed in $[16,17]$.

D-gap functions have been extended from VIs to (EP) as well. Indeed, the difference of two regularized gap functions

$$
\mathbf{p}_{\alpha \beta}(x):=\mathbf{p}_{\alpha}(x)-\mathbf{p}_{\beta}(x),
$$

with $0<\alpha<\beta$, is nonnegative on $\mathbb{R}^{n}$ and $\mathbf{p}_{\alpha \beta}\left(x^{*}\right)=0$ if and only if $x^{*}$ solves (EP). Thus, the global minima of $\mathbf{p}_{\alpha \beta}$ on $\mathbb{R}^{n}$ coincide with the solutions to (EP) (see $\left.[52,99]\right)$. The Dgap function inherits the differentiability properties of $\mathbf{p}_{\alpha}$ and $\mathbf{p}_{\beta}$ but in general is not convex. Stationary points of $\mathbf{p}_{\alpha \beta}$ coincide with the solutions to (EP) if the mappings $\nabla_{x} f(x, \cdot)+\nabla_{y} f(x, \cdot)$ are strictly monotone on $\mathbb{R}^{n}$ for any $x \in \mathbb{R}^{n}[99]$. Similarly to VIs, function $\sqrt{\overline{\mathbf{p}_{\alpha \beta}}}$ provides error bound results under suitable monotonicity assumptions on $f[23,101]$.

Several solution methods for (EP) are based on D-gap functions. Descent methods exploiting the direction $d=r(x)+\rho s(x)$, where $r(x)=y_{\alpha}(x)-y_{\beta}(x), s(x)=\alpha\left[x-y_{\alpha}(x)\right]-\beta\left[x-y_{\beta}(x)\right]$ and $\rho>0$ is small enough, have been introduced in [23, 52]. A descent method, which is similar to that proposed in [89] for VIs, is based on direction $d=y_{\alpha}(x)-y_{\beta}(x)$ and suitable updates of parameters $\alpha$ and $\beta$ [18]. Another descent method relies on the same direction $d=y_{\alpha}(x)-x$ which is exploited by the solution methods for $\mathbf{p}_{\alpha}[101]$.

The regularized Minty gap function (19) has been extended to (EP) in [86] and it has been used to develop an iterative method for solving strongly monotone equilibrium problems, while gap functions based on conjugate duality have been extended to (EP) in [2]. 


\subsection{An application to a class of Nash-Cournot equilibrium problems}

We now describe a problem of production competition over a network between several firms which produce the same commodity. We consider a modification of the oligopolistic model originally proposed in [59]. Given a transportation network $(\mathcal{N}, \mathcal{A})$, where $\mathcal{N}$ is the set of nodes and $\mathcal{A}$ the set of arcs, the firms and the markets are located at some subsets of nodes $I$ and $J$, respectively. Each firm $i \in I$ chooses the quantity $x_{j}^{i}$ to supply to each market $j \in J$ and the quantities $v_{a}^{i}$ to be sent on each $\operatorname{arc} a \in \mathcal{A}$. These variables are subject to flow-conservation constraints, i.e. for any $i \in I$ and $k \in \mathcal{N}$ we have

$$
\left(E v^{i}\right)_{k}= \begin{cases}-\sum_{j \in J} x_{j}^{i} & \text { if } k=i, \\ 0 & \text { if } k \notin J \\ x_{k}^{i} & \text { if } k \in J\end{cases}
$$

where $E$ is the node-arc incidence matrix of the network and $v^{i}=\left(v_{a}^{i}\right)_{a \in \mathcal{A}}$. Moreover, $q^{i}$ denotes the maximum quantity that firm $i$ may produce, i.e.

$$
\sum_{j \in J} x_{j}^{i} \leq q^{i}
$$

The goal of the firm $i$ is to maximize its profit given by

$$
\sum_{j \in J} x_{j}^{i} p_{j}\left(\sum_{\ell \in I} x_{j}^{\ell}\right)-\sum_{a \in \mathcal{A}} s_{a} v_{a}^{i}-\pi_{i}\left(\sum_{j \in J} x_{j}^{i}\right),
$$

where $p_{j}: \mathbb{R}_{+} \rightarrow \mathbb{R}_{+}$is the inverse demand function for market $j$, that is $p_{j}(z)$ denotes the unitary price at which the market $j$ requires a total quantity $z, s_{a}$ is the unitary transportation cost on arc $a$ and $\pi_{i}: \mathbb{R}_{+} \rightarrow \mathbb{R}_{+}$is the production cost function of firm $i$. Note that the first term of (38) depends on the quantities $x_{j}^{\ell}$ chosen by all the firms $\ell \in I$.

We say that an equilibrium state is reached when the flows and the quantities produced by the firms are such that no firm would increase its profit by changing its own choices while the other firms keep their own. This equilibrium definition coincides with the concept of Nash equilibrium in a noncooperative game where firms are the players and (38) are their payoff functions. Setting $x=\left(x_{j}^{i}\right)_{i \in I, j \in J}, v=\left(v^{i}\right)_{i \in I}$ and analogously $y$ and $w$, Nash equilibria of this game are the solutions of the abstract equilibrium problem (EP), where the bifunction $f$ is the Nikaido-Isoda function associated with the game [75], that is:

$$
\begin{array}{r}
f((x, v),(y, w))=\sum_{i \in I}\left[\sum_{j \in J} x_{j}^{i} p_{j}\left(\sum_{\ell \in I} x_{j}^{\ell}\right)-\sum_{j \in J} y_{j}^{i} p_{j}\left(y_{j}^{i}+\sum_{\ell \in I, \ell \neq i} x_{j}^{\ell}\right)\right. \\
\left.+\sum_{a \in \mathcal{A}} s_{a}\left(w_{a}^{i}-v_{a}^{i}\right)+\pi_{i}\left(\sum_{j \in J} y_{j}^{i}\right)-\pi_{i}\left(\sum_{j \in J} x_{j}^{i}\right)\right]
\end{array}
$$

and the feasible set $C$ is defined by constraints (36) and (37).

Example 5.1. Let us consider the transportation network in Fig. 6 , where $I=\{1,2\}, J=\{6,7\}$ and the number associated with each arc a denotes the unitary transportation cost $s_{a}$. 


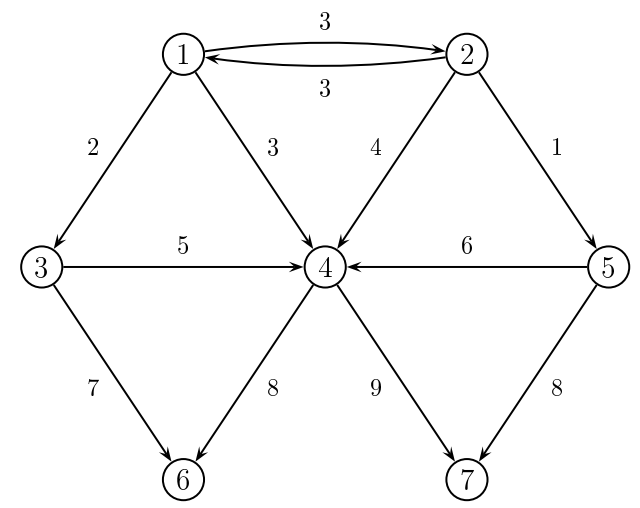

Figure 6: Transportation network in Example 5.1.

We assume that the production bounds are $q_{1}=70$ and $q_{2}=40$; that both markets have the same inverse demand function:

$$
p_{j}(z)=p(z):=\rho^{1 / \tau}(z+\sigma)^{-1 / \tau}, \quad j \in J,
$$

with $\rho=5000, \tau=1.1$ and $\sigma=0.01$ (see e.g. [69]), and that the production cost functions have the form

$$
\pi_{i}(z):=\gamma_{i} z+\left(1+\delta_{i}\right)^{-1} K_{i}^{-\delta_{i}} z^{1+\delta_{i}}, \quad i \in I,
$$

where parameters $\gamma_{i}, \delta_{i}$ and $K_{i}$ are reported in Table 2.

\begin{tabular}{c|ccc}
\hline$i$ & $\gamma_{i}$ & $\delta_{i}$ & $K_{i}$ \\
\hline 1 & 10 & $5 / 6$ & 5 \\
2 & 6 & 1 & 5 \\
\hline
\end{tabular}

Table 2: Parameters of cost functions $\pi_{i}$ in Example 5.1.

Since the functions $p$ and $\pi_{i}$ are convex and differentiable, the function $z \mapsto z p(z)$ is concave and the bifunction $f(\cdot,(y, w))$ is concave for any $(y, w)$. Therefore, condition (34) is fulfilled and the convergence of the modified descent algorithm proposed in [13] is guaranteed. We implemented this algorithm in MATLAB exploiting the built-in function fmincon from the Optimization Toolbox to evaluate the regularized gap function $\mathbf{p}_{\alpha}$ and to compute the search direction $y_{\alpha}(x)-x$. Table 3 reports the quantities supplied by each firm to each market in the first 10 iterations of the algorithm starting from a zero total production.

\section{Concluding remarks}

Merit functions have been introduced for a number of variational mathematical models. In this paper, we focused on three of the most important ones: variational inequalities, quasi-variational 


\begin{tabular}{c|rrrr|c}
\hline Iteration & $x_{6}^{1}$ & $x_{7}^{1}$ & $x_{6}^{2}$ & $x_{7}^{2}$ & $\mathbf{p}_{\alpha}(x)$ \\
\hline 0 & 0.0000 & 0.0000 & 0.0000 & 0.0000 & $1.0553 \mathrm{e}+04$ \\
1 & 11.5969 & 10.1858 & 11.3801 & 13.1382 & $1.1763 \mathrm{e}+03$ \\
2 & 29.5006 & 27.9329 & 19.5714 & 20.4286 & $8.7228 \mathrm{e}+00$ \\
3 & 32.6601 & 29.7497 & 18.8909 & 21.1091 & $8.6960 \mathrm{e}-02$ \\
4 & 32.5906 & 29.8771 & 18.6625 & 21.3375 & $2.5378 \mathrm{e}-03$ \\
5 & 32.5766 & 29.8855 & 18.6660 & 21.3340 & $1.5252 \mathrm{e}-03$ \\
6 & 32.5660 & 29.8917 & 18.6692 & 21.3308 & $9.1849 \mathrm{e}-04$ \\
7 & 32.5605 & 29.8950 & 18.6712 & 21.3288 & $6.5303 \mathrm{e}-04$ \\
8 & 32.5560 & 29.8977 & 18.6730 & 21.3270 & $4.6451 \mathrm{e}-04$ \\
9 & 32.5534 & 29.8992 & 18.6742 & 21.3258 & $3.6820 \mathrm{e}-04$ \\
10 & 32.5511 & 29.9006 & 18.6753 & 21.3247 & $2.9189 \mathrm{e}-04$ \\
\hline
\end{tabular}

Table 3: Numerical results of the modified descent algorithm proposed in [13] applied to Example 5.1 .

inequalities and abstract equilibrium problems. Among others relevant models we recall setvalued variational inequalities, vector variational inequalities and generalized Nash equilibrium problems.

The merit function approach has been extensively developed for VIs in the last two decades, while it is still at a quite early stage for more general problems. We believe that this is partially due to the complexity of these problems, but above all because many real-world applications of these problems have arisen recently. Therefore, there are still many challenging open problems regarding merit functions which are worthy of being investigated.

Merit functions for QVIs need to be further investigated: for instance, the general condition under which the stationary points of the regularized gap function are solutions to (QVI) should be deepened for different classes of problems according to the set-valued mapping defining the feasible region. Furthermore, to the best of our knowledge no ad-hoc descent method based on merit functions has been developed so far.

Regarding abstract equilibrium problems, the convergence of descent methods developed for them is usually based on differentiability assumptions. We think that some efforts should be devoted to develop algorithms for nonsmooth problems, which include nonsmooth Nash equilibrium problems as special cases. Moreover, it would be interesting to extend the Moreau-Yosida regularization to merit functions for abstract equilibrium problems. Finally, we believe that new merit functions could be developed without assuming the convexity of $f(x, \cdot)$ : this might allow to extend the merit function approach to nonconvex Nash equilibrium problems.

\section{References}

[1] Altangerel L, Bot RI, Wanka G (2007) On the construction of gap functions for variational inequalities via conjugate duality. Asia Pac J Op Res 24: 353-371

[2] Altangerel L, Bot RI, Wanka G (2006) On gap functions for equilibrium problems via Fenchel duality. Pac J Optim 2: 667-678 
[3] Altman E, Wynter L (2004) Equilibrium, games, and pricing in transportation and telecommunication networks. Netw Spat Econ 4: 7-21

[4] Anselmi J, Ardagna D, Passacantando M (2013) Generalized Nash equilibria for SaaS/PaaS clouds. European J Oper Res, doi: 10.1016/j.ejor.2013.12.007

[5] Ardagna D, Panicucci B, Passacantando M (2011) A game theoretic formulation of the service provisioning problem in cloud systems. In Proceedings of the 20th international conference on World Wide Web, Hyderabad, India: 177-186

[6] Ardagna D, Panicucci B, Passacantando M (2013) Generalized Nash equilibria for the service provisioning problem in cloud systems. IEEE T Serv Comput 6: 429-442

[7] Auchmuty G (1989) Variational principles for variational inequalities. Numer Funct Anal Optim 10: 863-874

[8] Auslender A (1976) Optimisation. Méthodes numériques. Masson, Paris

[9] Aussel D, Correa R, Marechal M (2011) Gap functions for quasivariational inequalities and generalized Nash equilibrium problems. J Optim Theory Appl 151: 474-488

[10] Baiocchi C, Capelo A (1984) Variational and quasivariational inequalities: applications to free boundary problems. Wiley, New York

[11] Bensoussan A, Goursat M, Lions J-L (1973) Contrôle impulsionnel et inéquations quasivariationnelles stationnaires. C R Acad Sci Paris Sér A 276: 1279-1284

[12] Bensoussan A, Lions J-L (1973) Nouvelle formulation de problèmes de contrôle impulsionnel et applications. C R Acad Sci Paris Sér A 276: 1189-1192

[13] Bigi G, Castellani M, Pappalardo M (2009) A new solution method for equilibrium problems. Optim Methods Softw 24: 895-911

[14] Bigi G, Castellani M, Pappalardo M, Passacantando M (2013) Existence and solution methods for equilibria. European J Oper Res 227: 1-11

[15] Bigi G, Panicucci B (2010) A successive linear programming algorithm for nonsmooth monotone variational inequalities. Optim Methods Softw 25: 29-35

[16] Bigi G, Passacantando M (2012) Gap functions and penalization for solving equilibrium problems with nonlinear constraints. Comput Optim Appl 53: 323-346

[17] Bigi G, Passacantando M (2013) Descent and penalization techniques for equilibrium problems with nonlinear constraints. J Optim Theory Appl. doi: 10.1007/s10957-013-0473-7

[18] Bigi G, Passacantando M (2013) D-gap functions and descent techniques for solving equilibrium problems. Technical Report TR-13-15 del Dipartimento di Informatica - University of Pisa

[19] Blum E, Oettli W (1994) From optimization and variational inequalities to equilibrium problems. Math Stud 63: 123-145 
[20] Cavazzuti E, Pappalardo M, Passacantando M (2002) Nash equilibria, variational inequalities, and dynamical systems. J Optim Theory Appl 114: 491-506

[21] Chadli O, Konnov IV, Yao J-C (2004) Descent methods for equilibrium problems in a Banach space. Comput Math Appl 48: 609-616

[22] Chan D, Pang J-S (1982) The generalized quasi-variational inequality problem. Math Oper Res 7: 211-222

[23] Cherugondi C (2013) A note on D-gap functions for equilibrium problems. Optimization 62: $211-226$

[24] Dafermos S (1980) Traffic equilibrium and variational inequalities. Transportation Sci 14: $42-54$

[25] Danskin JM (1966) The theory of max-min, with applications. SIAM J Apple Math 14: 641-664

[26] Di Lorenzo D, Passacantando M, Sciandrone M (2013) A convergent inexact solution method for equilibrium problems. Optim Methods Softw. doi: 10.1080/10556788.2013.796376

[27] Dietrich H (2001) Optimal control problems for certain quasivariational inequalities. Optimization 49: 67-93

[28] Drouet L, Haurie A, Moresino F, Vial J-P, Vielle M, Viguier L (2008) An oracle based method to compute a coupled equilibrium in a model of international climate policy. Comput Manag Sci 5: 119-140

[29] Eaves BC (1971) On the basic theorem of complementarity. Math Program 1: 68-75

[30] Facchinei F, Kanzow C (2007) Generalized Nash equilibrium problems. 4OR 5: 173-210

[31] Facchinei F, Kanzow C, Sagratella S (2013) Solving quasi-variational inequalities via their KKT conditions. Math Program. doi:10.1007/s10107-013-0637-0

[32] Facchinei F, Kanzow C, Sagratella S (2013) QVILIB: A library of quasi-variational inequality test problems. Pac J Optim 9: 225-250

[33] Facchinei F, Pang J-S (2003) Finite-dimensional variational inequalities and complementarity problems. Springer, New York

[34] Ferris MC, Pang J-S (1997) Engineering and economic applications of complementarity problems. SIAM Rev 39: 669-713

[35] Fischer A, Jang H (2001) Merit functions for complementarity and related problems: a survey. Comput Optim Appl 17: 159-182

[36] Forgó F, Fülöp J, Prill M (2005), Game theoretic models for climate change negotiations. European J Oper Res 160: 252-267

[37] Fukushima M (1992) Equivalent differentiable optimization problems and descent methods for asymmetric variational inequality problems. Math Program 53: 99-110 
[38] Fukushima M (2007) A class of gap functions for quasi-variational inequality problems. J Ind Manag Optim 3: 165-171

[39] Giannessi F (1995) Separation of sets and gap functions for quasi-variational inequalities. In Variational Inequalities and Network Equilibrium Problems, F.Giannessi and A.Maugeri (eds), Plenum: 101-121

[40] Giannessi F (1998) On Minty variational principle. In F. Giannessi, S. Komlósi, T. Rapcsák Eds., New trends in mathematical programming, Kluwer Academic Publishers: 93-99

[41] Gupta R, Mehra A (2012) Gap functions and error bounds for quasivariational inequalities. J Glob Optim 53: 737-748

[42] Harker PT, Pang J-S (1990) Finite-dimensional variational inequalities and nonlinear complementarity problem: a survey of theory, algorithms and applications. Math Program 48: $161-220$

[43] Harms N, Kanzow C, Stein O (2013) Smoothness properties of a regularized gap function for quasi-variational inequalities. Optim Methods Softw. doi: 10.1080/10556788.2013.841694

[44] Hartman P, Stampacchia G (1966) On some nonlinear elliptic differential functional equations. Acta Math 115: 153-188

[45] Huang LR, Ng KF (2005) Equivalent optimization formulations and error bounds for variational inequality problems. J Optim Theory Appl 125: 299-314

[46] Kanzow C, Fukushima M (1998) Theoretical and numerical investigation of the D-gap function for box constrained variational inequalities. Math Program 83: 55-87

[47] Kanzow C, Fukushima M (1998) Solving box constrained variational inequalities by using the natural residual with D-gap function globalization. Oper Res Lett 23: 45-51

[48] Konnov IV (2007) On variational inequalities for auction market problems. Optim Lett 1: $155-162$

[49] Konnov IV (2008) Variational inequalities for modeling auction markets with price mappings. Open Oper Res J 2: 29-37

[50] Konnov IV (2008) Spatial equilibrium problems for auction-type systems. Russian Math 52: $30-44$

[51] Konnov IV, Pinyagina OV (2003) Descent method with respect to the gap function for nonsmooth equilibrium problems. Russian Math 47: 67-73

[52] Konnov IV, Pinyagina OV (2003) D-gap functions for a class of equilibrium problems in Banach spaces. Comput Methods Appl Math 3: 274-286

[53] Larsson T, Patriksson M (1994) A class of gap functions for variational inequalities. Math Program 64: 53-79 
[54] Liu Z, Nagurney A (2007) Financial networks with intermediation and transportation network equilibria: a supernetwork equivalence and reinterpretation of the equilibrium conditions with computations. Comput Manag Sci 4: 243-281

[55] Li G, Tang C, Wei Z (2010) Error bound results for generalized D-gap functions of nonsmooth variational inequality problems. J Comput Appl Math 233: 2795-2806

[56] Li G, Ng KF (2009) Error bounds of generalized D-gap functions for nonsmooth and nonmonotone variational inequality problems. SIAM J Optim 20: 667-690

[57] Mangasarian OL, Solodov MV (1993) Nonlinear complementarity as unconstrained and constrained minimization. Math Program 2: 277-297

[58] Marcotte P (1985) A new algorithm for solving variational inequalities with applications to traffic assignment problem. Math Program 33: 339-351

[59] Marcotte P (1987) Algorithms for the network oligopoly problem. J Oper Res Soc 38: 10511065

[60] Marcotte P, Dussault JP (1987) A note on globally convergent Newton method for solving monotone variational inequalities. Oper Res Lett 6: 273--284

[61] Marcotte P, Dussault JP (1989) A sequential linear programming algorithm for solving monotone variational inequalities. SIAM J Optim 27: 1260-1278

[62] Marcotte P, Zhu D (1998) Weak sharp solutions of variational inequalities. SIAM J Optim 9: $179--189$

[63] Mastroeni G (1994) Some relations between duality theory for extremum problems and Variational Inequalities. Le Matematiche 49: 295-304

[64] Mastroeni G (2005) Gap functions and descent methods for Minty variational inequality. In Optimization and control with applications, Springer: 529-547

[65] Mastroeni G (2003) Gap functions for equilibrium problems. J Global Optim 27: 411-426

[66] Miller N, Ruszczynski A (2008) Risk-adjusted probability measures in portfolio optimization with coherent measures of risk. European J Oper Res 191: 193-206

[67] Minty GJ (1967) On the generalization of a direct method of the calculus of variations. Bull Amer Math Soc 73: 315-321

[68] Mordukhovich BS, Outrata JV, Cervinka M (2007) Equilibrium problems with complementarity constraints: case study with applications to oligopolistic markets. Optimization 56: 479-494

[69] Murphy FH, Sherali HD, Soyster AL (1982) A mathematical programming approach for determining oligopolistic market equilibrium. Math Program 24: 92-106

[70] Nagurney A (1993) Network economics: a variational inequality approach. Kluwer, Dordrecht 
[71] Nagurney A (2010) Formulation and analysis of horizontal mergers among oligopolistic firms with insights into the merger paradox: a supply chain network perspective. Comput Manag Sci 7: $377-406$

[72] Ng KF, Tan LL (2007) D-gap functions for nonsmooth variational inequality problems. J Optim Theory Appl 133: 77-97

[73] Ng KF, Tan LL (2007) Error bounds of regularized gap functions for nonsmooth variational inequality problems. Math Program 110: 405-429

[74] Nguyen S, Dupuis C (1984) An efficient method for computing traffic equilibria in networks with asymmetric transportation costs. Transp Sci 18: 185-202

[75] Nikaido H, Isoda K (1955), Note on noncooperative convex games. Pacific J Math 5: 807-815

[76] Pang J-S, Scutari G, Palomar DP, Facchinei F (2010) Design of cognitive radio systems under temperature-interference constraints: a variational inequality approach. IEEE T Signal Proces 58: 3251-3271

[77] Pappalardo M, Passacantando M (2002) Stability for equilibrium problems: from variational inequalities to dynamical systems. J Optim Theory Appl 113: 567-582

[78] Pappalardo M, Passacantando M (2004) Gap functions and Lyapunov functions. J Global Optim 28: 379-385

[79] Panicucci B, Pappalardo M, Passacantando M (2009) A globally convergent descent method for nonsmooth variational inequalities. Comput Optim Appl 43: 197-211

[80] Patriksson M (1994) The traffic assignment problem: models and methods. VSP, Utrecht

[81] Peng J-M (1997) Equivalence of variational inequality problems to unconstrained minimization. Math Program 78: 347-355

[82] Peng J-M, Kanzow C, Fukushima M (1999) A hybrid Josephy-Newton method for solving box constrained variational inequality problems via the D-gap function. Optim Methods Softw 10: $687-710$

[83] Peng J-M, Yuan Y (1997) Unconstrained methods for generalized complementarity problems. J Comput Math 15: 253-264

[84] Peng J-M, Fukushima M (1999) A hybrid Newton method for solving the variational inequality problem via the D-gap function. Math Program 86: 367-386

[85] Qu B, Wang CY, Zhang JZ (2003) Convergence and error bound of a method for solving variational inequality problems via the generalized D-gap function. J Optim Theory Appl 119: $535-552$

[86] Quoc TD, Muu LD (2012) Iterative methods for solving monotone equilibrium problems via dual gap functions. Comput Optim Appl 51: 709-728

[87] Rockafellar RT (1970) Convex analysis. Princeton University Press, Princeton 
[88] Scutari G, Palomar DP, Barbarossa S (2010) Competitive optimization of cognitive radio MIMO systems via game theory. In: Convex optimization in signal processing and communications, Cambridge University Press, Cambridge: 387-442

[89] Solodov MV, Tseng P (2000) Some methods based on the D-gap function for solving monotone variational inequalities. Comput Optim Appl 17: 255-277

[90] Sun D, Fukushima M, Qi L (1997) A computable generalized Hessian of the D-gap function and Newton-type methods for variational inequality problems. In Complementarity and variational problems, SIAM: $452-473$

[91] Taji K (2008) On gap functions for quasi-variational inequalities. Abstr Appl Anal, Art. ID 531361, 7 pp.

[92] Taji K, Fukushima M (1996) A new merit function and a successive quadratic programming algorithm for variational inequality problems. SIAM J Optim 6: 704-713

[93] Taji K, Fukushima M, Ibaraki T (1993) A globally convergent Newton method for solving strongly monotone variational inequalities. Math Program 58: 369-383

[94] Tan LL (2007) Regularized gap functions for nonsmooth variational inequality problems. J Math Anal Appl 334: 1022-1038

[95] Wardrop JG (1952) Some theoretical aspects of road traffic research. Proceedings of the Institute of Civil Engineers, 1: 325-378

[96] Wu JH, Florian M, Marcotte P (1993) A general descent framework for the monotone variational inequality problem. Math Program 61: 281-300

[97] Yamashita N, Fukushima M (1997) Equivalent unconstrained minimization and global error bounds for variational inequality problems. SIAM J Contr Optim 35: 273-284

[98] Yamashita N, Taji K, Fukushima M (1997) Unconstrained optimization reformulations of variational inequality problems. J Optim Theory Appl 92: 439-456

[99] Zhang L, Han JY (2009) Unconstrained optimization reformulations of equilibrium problems. Acta Math Sin 25: 343-354

[100] Zhang J, Wan C, Xiu N (2003) The dual gap function for variational inequalities. Appl Math Optim 48: 129-148

[101] Zhang L, Wu S-Y (2009) An algorithm based on the generalized D-gap function for equilibrium problems. J Comput Appl Math 231: 403-411

[102] Zhao L, Nagurney A (2008) A network equilibrium framework for internet advertising: models, qualitative analysis, and algorithms. European J Oper Res 187: 456-472

[103] Zhu DL, Marcotte P (1993) Modified descent methods for solving the monotone variational inequality problem. Oper Res Lett 14: 111-120

[104] Zhu DL, Marcotte P (1994) An extended descent framework for variational inequalities. J Optim Theory Appl 80: 349-366 